\title{
AN EVALUATION OF ACCOUNTING-BASED FINDING COSTS AS EFFICIENCY \\ MEASURES FOR OIL AND GAS EXPLORATION
}

Charles E. Boynton, IV

Jeffery P. Boone

\author{
Institute of Petroleum Accounting \\ University of North Texas \\ Denton, Texas
}

\section{DISCLAIMER}

This report was prepared as an account of work sponsored by an agency of the United States Government. Neither the United States Government nor any agency thereof, nor any of their employees, makes any warranty, express or implied, or assumes any legal liability or responsibility for the accuracy, completeness, or usefulness of any information, apparatus, product, or process disclosed, or represents that its use would not infringe privately owned rights. Reference herein to any specific commercial product, process, or service by trade name, trademark, manufacturer, or otherwise does not necessarily constitute or imply its endorsement, recommendation, or favoring by the United States Government or any agency thereof. The views and opinions of authors expressed herein do not necessarily state or reflect those of the United States Government or any agency thereof. 


\section{DISCLAIMER}

Portions of this document may be illegible in electronic image products. Images are produced from the best available original document. 
AN EVALUATION OF ACCOUNTING-BASED FINDING COSTS AS EFFICIENCY MEASURES FOR OIL AND GAS EXPLORATION

\section{Executive Summary}

We operationalized firm-specific exploration efficiency as the difference between a firm-specific intercept estimated in a fixed-effects panel data Cobb-Douglas production frontier model and the maximum firm-specific intercept estimated in that model. The production model was estimated during two different time periods, 1982-1985 and 1989-1992, allowing efficiency to vary intertemporally. This efficiency estimate served as a benchmark against which we compared various measures of inverse finding costs. We assumed that the degree of association with an efficiency benchmark is an important attribute of any finding cost measure and that, further, the degree of association may be used as a metric for choosing between alternative finding cost measures. Accordingly, we evaluated the cross-sectional statistical association between estimated efficiency and alternative inverse finding cost measures.

We discovered that the inverse finding cost measure that exhibited the strongest association with efficiency during the two time periods was a three-year moving- average finding cost which included exploration plus development expenditures as costs and reserve extensions and additions plus revisions as the units added. This result is interesting for three reasons. First, our results lend empirical support for the common practice of reporting three-year or five-year moving-average finding costs in 
addition to single year. Second, our results lend empirical support for the argument that exploration plus development expenditures is a better measure of reserve replacement cost than exploration expenditures alone. Third, results add further documentation of an anomaly we reported a year ago in our preliminary report: Inverse finding cost measures computed with reserve revisions exhibit stronger statistical association with efficiency than those measures that exclude reserve revisions. This finding is anomalous because reserve revisions should represent random estimation errors and, therefore, would be expected to weaken the association rather than strengthen the association. That reserve revisions strengthen the association suggests that reserve revisions contain a systematic component and are not merely random estimation errors.

Finally, our study presented efficiency measures for 66 firms that survived the tumultuous decade of the 1980s, 146 firms that did not survive the decade, and 53 firms that entered the industry during the 1980s. The results suggest that survivor firms were more efficient than non-survivors or new firms, and that non-survivor firms were least efficient among the three groups. The results also show that the relative dispersion in efficiency measures decreased during the period 1989-1992 as compared to the relative dispersion during the period 1982-1985, suggesting that industry restructuring produced a more homogeneous set of firms. 
AN EVALUATION OF ACCOUNTING-BASED FINDING COSTS AS EFFICIENCY MEASURES FOR OIL AND GAS EXPLORATION

\section{Introduction}

This study presents empirical evidence intended to answer two related research questions. The first question asks whether finding cost measures developed from publicly available accounting data accurately assess the relative exploration efficiency of oil and gas firms. The question arises because experts in financial analysis disagree about the potential usefulness of the finding cost measure as an assessment tool. Answering this question is important because financial statement users are believed increasingly to use accounting-based finding costs to assess relative exploration efficiency.

The second question asks which types of accounting data produce a finding cost measure that constitutes the best gauge of relative exploration efficiency. The question arises because there exist several different ways of computing finding costs, and it remains an open question as to which measure, if any, is most strongly associated with exploration efficiency. Answering this question will provide helpful information to those who wish to assess the relative exploration efficiency of oil and gas firms using publicly available accounting information.

We estimate a fixed-effects panel data Cobb-Douglas production frontier model with heterogeneity in intercepts that is invariant within estimation periods (1982-1985, 1989-1992) but varies between estimation periods. These intercepts measure each 
firm's productivity, and firm-specific efficiency is assessed relative to the estimated production frontier. The resulting efficiency measures are cross-sectionally correlated with various accounting-based inverse finding cost measures to determine which finding cost measure, if any, exhibits the strongest association with firm-specific efficiency. The analysis found that accounting-based inverse finding cost measures are strongly associated with exploration efficiency. In particular, a threeyear moving-average finding cost which includes development expenditures and reserve revisions appears to dominate all others in terms of association with efficiency.

While not our principal focus, the research design also allowed us to explore how the relative efficiency of firms changed between the two estimation periods and to compare the mean efficiency of survivor firms to nonsurvivor firms and new entrants. The analysis showed that the mean efficiency measure of survivor firms was greater than both nonsurvivor firms and new entrants. The relative dispersion in the efficiency measure was greater during the 1982-1985 time period than during the 19891992 time period while the mean efficiency measure was greater during the later period, suggesting that the restructuring in the extractive petroleum industry during the 1980s eliminated less efficient firms and produced a more homogenous group of firms. The results provide reasonable and believable evidence on the pattern of efficiency changes that occurred during a period of widespread industry restructuring. 


\section{Motivation}

The issue of whether average finding costs computed from publicly available financial statement data provide useful information about exploration efficiency, profitability, and future cash flows of an oil and gas firm is unsettled. These finding costs typically are computed by dividing the units of oil and gas discovered (with gas converted to a barrel of oil equivalent unit) into the costs incurred to "find" those units.

The conventional view that finding costs provide useful information is articulated by a recent textbook on financial statement analysis (White, Sondhi, and Fried 1994, p. 483), whose authors write that "While periodic finding costs are volatile, over longer time periods they provide evidence as to management's proficiency at the discovery of reserves." Echoing this view, the director of stock research at salomon Brothers, Inc. argues that "After reserve replacement ratios, finding costs are the most critical internal factor for the oil companies" (Randol 1992, p.17).

Other analysts are less persuaded that finding costs provide useful information about an oil and gas company. Mayer (1992, p. 29) writes that

Wall street performs a big disservice to the institutional investment community by placing undeserved emphasis on reserve replacement ratios and finding costs as measures of how well a company has done. Oil companies do not pay particular attention to these numbers, and no major oil company bases its own internal economics on them.

Firms' and industry-wide average finding costs computed from publicly available financial statement data increasingly are 
cited, compared, and discussed with little question about their relevance. Juxtaposed against the increased reporting of finding costs (and, presumably the increased reliance placed on the measure by those asking for or issuing the reports), the disagreement among financial analysts concerning the usefulness of finding costs underscores the need for empirical research to evaluate the usefulness of the measure. We are aware of no study that has rigorously evaluated the potential usefulness of accounting-based finding costs computed from publicly available financial statement data.

Whether or not finding costs computed from publicly available financial statement data are useful is not the only important unsettled issue concerning finding costs. A second issue concerns how to compute finding costs. Among the key conceptual issues identified by Gaddis, Brock and Boynton (1992) are what 1) costs to use, 2) reserves to use, and 3) time period to use.

An important dimension of the cost issue concerns whether development costs should be included in the finding cost measure. Those opposing such inclusion argue that finding costs are intended to gauge exploration activity so that the finding cost measure should include exploration costs but not development costs. Others argue that the cost of developing reserves is actually a dimension of the effort to replace reserves and so the average finding cost measures should include development costs. 
The reserve revision issue concerns whether to include revision of reserve estimates as part of reserve additions, while the time period issue is concerned with whether single-year or multiple-year finding costs are most appropriate. The usual argument advanced is that a lag structure exists between exploration spending and reserve additions, rendering single-year finding costs measures meaningless and multiple-year finding cost measures necessary. Three-year moving average finding cost measures are often advocated as a useful alternative to singleyear measures.

In summary, our study is motivated by the disagreement among financial analysts concerning the 1) potential usefulness of finding costs as an assessment tool and 2) the relevant costs, reserves, and time periods to be included within the measure.

\section{Research Design}

Model

our model is motivated by the work of Cornwell, Schmidt, and Sickles (1990) and Schmidt and Sickles (1984) who propose using panel data to estimate frontier production models. In their model, the intercept varies across firms, and differences in the intercept are interpreted as variation in productivity levels (or technical efficiency). The attractive feature of their model is that no strong distributional assumptions are required about technical efficiency, and no assumption of independence between technical efficiency and the explanatory variables is needed. 
Reiss (1990) proposes a Cobb-Douglas reserve addition discovery function where reserve additions are a function of four factor inputs - exploratory wells drilled, development wells drilled, proved leaseholdings, and undeveloped leaseholdings. We use this reserve discovery function but propose a more parsimonious set of explanatory variables. Specifically, we propose to use current and lagged exploration and development spending as indices for the quantity of input factors used in reserve discovery. We also include production volume as an index of all other oil and gas activities, plant property and equipment, and labor and energy consumption. Our reserve discovery function ( $\log$ transformed) is

\section{(1) Model 1}

$$
\ln A D D=\ln \alpha_{i}+\beta_{1} L N E X P+\beta_{2} L N D E V+\beta_{3} L N O U T+\beta_{4} L A G E X P+\beta_{5} L A G D E V+\varepsilon
$$

where

\begin{tabular}{|c|c|c|}
\hline LNEXP & $=$ & $\begin{array}{l}\text { natural } \log \text { of worldwide exploration } \\
\text { expenditures }\end{array}$ \\
\hline LNDEV & $=$ & $\begin{array}{l}\text { natural log of worldwide development } \\
\text { expenditures }\end{array}$ \\
\hline INOUT & $=$ & $\begin{array}{l}\text { natural log of worldwide oil and gas } \\
\text { production measured in barrel-of-oil } \\
\text { equivalent units (BOE), with } 6 \text { mef of natural } \\
\text { gas equal to } 1 \mathrm{BOE} \text {. }\end{array}$ \\
\hline LAGEXP & $=$ & one year lagged LNEXP \\
\hline
\end{tabular}




$$
\begin{aligned}
\text { LAGDEV }= & \text { one year lagged LNDEV } \\
\text { LNADD }= & \text { natural log of worldwide oil and gas reserve } \\
& \text { additions and extensions measured in BOE } \\
& \text { units. }
\end{aligned}
$$

The frontier intercept $\alpha$ is estimated as $\hat{\alpha}=\max _{i}\left(\ln \hat{\alpha}_{i}\right)$, and the level of efficiency for firm $i$ (EFF) is $\ln \hat{\alpha}_{i}-\ln \hat{\alpha}$. We estimate model 1 as a fixed-effects least squares dummy variable model with n-1 firm dummies, so that 1 n $\hat{\alpha}_{i}$ is computed as the sum of the estimated model intercept plus the estimated parameter for the firm dummy.

We estimate model 1 separately for the period 1982-1985 and the period 1989-1992 to permit firms' productivity levels to change during these two very different periods of oil and gas exploration activity. We assume that the years 1986-1987 were transition years between the exploration environments that characterized the two periods and therefore omit these years from the analysis. Data for 1981 and 1988 are used to provide lags for 1982 and 1989. We therefore use data from 10 of the 12 years in 1981-1992 omitting only the center two years, 1986 and 1987.

\section{Sample}

our sample consists of firms for which uninterrupted observations on the variables were available in the Arthur Andersen Reserve Disclosure Database during either the period 1981-1985 or 1988-1992. This data source collects data on the oil and gas exploration activities disclosed by publicly held oil 
and gas firms pursuant to statement of Financial Accounting Standards No. 69 .

The data screen produced three groups of firms. Group A consisted of 66 firms for which data were available during both the 1981-1985 period and the 1989-1992 period. Group B consisted of 146 firms for which data were available during the 1981-1985 period but were unavailable during the period 1985-1992. Group C consisted of 53 firms for which data were available during the period 1985-1992 but unavailable during the period 1982-1985. In general, firms within Group B were firms that merged, liquidated, or ceased oil exploration. We refer to Group B firms as "Nonsurvivors". In general, Group C consists of new firms or reorganized firms, so we call these firms "New Entrants". We term Group A firms as "Survivors" for obvious reasons. Descriptive statistics on the sample are presented in Table 1.

\begin{tabular}{|l|c|c|c|c||}
\hline \multicolumn{5}{|c|}{ Table 1.--Descriptive statistics on sample firms. } \\
\hline & \multicolumn{2}{|c|}{ Group A } & Group B & Group C \\
\cline { 2 - 5 } & $1982-1985$ & $1988-1992$ & $1982-1985$ & $1989-1992$ \\
\hline $\begin{array}{l}\text { Exploration } \\
\text { expenditures } \\
\text { (millions of } \\
\text { dollars) }\end{array}$ & $\$ 46,826$ & $\$ 30,064$ & $\$ 15,199$ & $\$ 21,183$ \\
\hline $\begin{array}{l}\text { Reserves } \\
\text { added } \\
\text { (BoE units) }\end{array}$ & 8,252 & 9,167 & 3,721 & 8,514 \\
\hline
\end{tabular}




\section{Empirical Results}

We pool Group A and Group B firms to form a panel dataset consisting of four annual observations (1982-1985) with one-year lags on 212 firms, and pool Group $A$ and Group $C$ firms to form a second panel dataset consisting of four annual observations (1989-1992) with one-year lags on 119 firms. To facilitate discussion, we refer to 1982-1985 and 1989-1992 as the early and late periods, respectively. Model $I$ is separately estimated across pooled Group $A / B$ and across pooled Group $A / C$ and efficiency measures computed. The parameter estimates of Model 1 appear in Appendix A (printing of firm dummies was suppressed) . Comparing the Model 1 parameters estimated across 1982-1985 with the parameters estimated across 1989-1992 shows that both LNEXP and LNDEV are strongly significant in both periods, while neither LNOUT, IAAGEXP, and LAGDEV are significant in either period. The estimated parameters show that a $1 \%$ increase in current exploration spending during $1982-1985$ is associated with a $0.18 \%$ increase in current reserve additions, while a $1 \%$ increase in current exploration spending during 1989-1992 is associated with a $0.33 \%$ increase in current reserve additions. Similarly, a $1 \%$ increase in development spending during the early period is associated with $0.34 \%$ increase in current reserve additions while a similar increase during the later period is associated with a $0.50 \%$ increase in reserve quantities. It is interesting to note that the estimated elasticities for exploration and development spending in the early period are very 
similar to the 0.11 and 0.44 (for exploration and development wells, respectively) reported by Reiss (1990) for a similar time period.

We tested the null hypothesis that exploration and development exhibit constant returns to scale by testing whether the estimated elasticities for LNEXP, LNDEV, and LNOUT sum to 1. The data were able to reject the null hypothesis in the early period but were unable to reject the null hypothesis in the later period. Because Reiss (1990) is unable to reject the null hypothesis of constant returns to scale during a time period that roughly corresponds to our early period, the issue of potential model misspecification arises. Our concern is not with whether a correlated omitted variable has biased the estimated elasticities. Rather, we are concerned with the effect an omitted variable might have on the estimated firm-specific intercepts since we are principally concerned with using these parameters from the model to estimate efficiency. More specifically, if a consistent estimate of the firm-specific intercept is given as

$$
\hat{\alpha}^{*}{ }_{i}=\overline{R A}-\hat{\beta}^{\prime} \bar{X}+\theta^{\prime} \bar{Z}
$$

but the estimated firm-specific intercept is

$$
\hat{\alpha}_{i}=\overline{R A}-\beta^{\prime} \bar{X}
$$


then the estimated firm-specific intercept will be equal to $\hat{\alpha}^{*}{ }_{i}+\theta^{\prime} \mathrm{z}$ (assuming $\mathrm{z}$ is uncorrelated with the explanatory variables in the $\mathrm{x}$ vector). In particular, if $\mathrm{z}$ is correlated with size then the estimated efficiencies, discussed below, could be attributed to size rather than to any underlying efficiency.

To evaluate whether the low estimated elasticities are attributable to a size-related omitted variable, we expand model 1 to include LNTRB - a size proxy operationalized as the natural log of beginning oil and gas reserves measured in BOE units. This model is labeled Model 2 in Appendix A. LNTRB is statistically significant in the early period but not in the later period, suggesting the possibility of size-related bias in the efficiency estimates from Model 1 during the early period. Consequently, we will test the robustness of our efficiency analysis from Model 1 by using firm-specific intercepts from Model 2 as well.

Appendix B presents the firm-specific efficiency measures (EFF) computed from Model 1 as EFF $=\ln \hat{\alpha}_{i}-\max _{i}\left(\ln \hat{\alpha}_{i}\right)$. We computed the mean EFF for firms in Group $A$ and compared to the mean EFF computed for firms in Group B and Group $C$. These results are reported in Appendix C. Two results are especially noteworthy. First, notice that the mean EFF in Group A during the early period $(.147)$ is greater than the mean EFF in Group B $(.068)$, with the null hypothesis of equal means rejected at the 0.0001 level of significance. Similarly, the mean EFF in Group A during the late period $(.283)$ is also greater than the mean EFF 
in Group C (.254), but the difference is not statistically significant. Second, the relative dispersion in EFF as measured by the coefficient of variation shows much less dispersion in the later period $(\mathrm{CV}=69.18)$ than in the earlier $(\mathrm{CV}=122.28)$, suggesting that oil and gas firms not only have increased their overall level of efficiency but also are less dissimilar in terms of their level of efficiency - that is, firms are more homogenous in the later period.

While the level of and change in these efficiency measures is interesting in its own right, analysis of these measures is not our principal focus. These efficiency measures are simply a means to an end - they give us a benchmark against which we can compare alternative measures of average finding costs.

We operationalize eight different measures of average finding costs. Each definition represents a cell within a $2 \times 2 \times 2$ matrix that is defined by whether 1 ) only exploration costs or both exploration and development costs are included in the numerator, 2) reserve revisions are or are not included in the denominator, and 3) whether finding costs are computed for a single year or as a moving average across 3 years. As a practical matter, we use the inverse of these finding costs in our analysis because reserve additions per dollar expended should be positively correlated with exploration efficiency, while dollars expended per unit of reserve additions should be negatively correlated with exploration efficiency. Table 2 summarizes these eight inverse finding cost measures. 


\begin{tabular}{|c|c|}
\hline Variable & Definition \\
\hline IFC1 & $1 /\left[\right.$ WWEXPL $_{t} /$ WWBOEEXT $\left._{t}\right]$ \\
\hline IFC3 & $1 /\left[\sum_{1=0}^{2} \operatorname{WWEXPL}_{\mathrm{t} \cdot 1} / \sum_{1=0}^{2} \mathrm{WWBOEEXT}_{\mathrm{t}-1}\right]$ \\
\hline IFCIR & $1 /\left[\right.$ WWEXPL $_{t} /\left(\right.$ WWBOEEXT $_{t}+$ WWBOEREV $\left.\left._{t}\right)\right]$ \\
\hline IFC3R & $I /\left[\sum_{1=0}^{2}\right.$ WWEXPL $_{t-1} / \sum_{1=0}^{2}$ WWBOEEXT $_{t-1}+$ WWBOEREV $\left._{t-1}\right]$ \\
\hline IFCID & $1 /\left[\left(W^{2} \mathrm{EXPL}_{t}+\mathrm{WWDEV}_{t}\right) /\right.$ WWBOEEXT $\left._{t}\right)$ \\
\hline IFC3D & 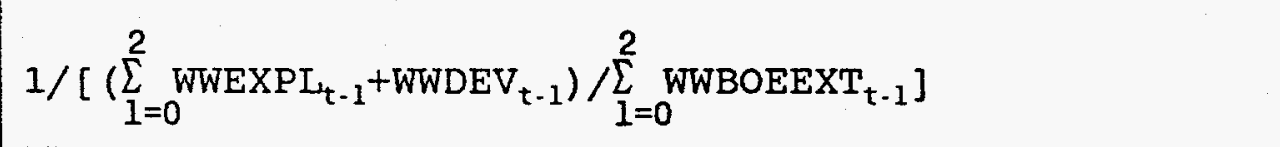 \\
\hline IFC1RD & $1 /\left[\left(\right.\right.$ WWEXPL $_{t}+$ WWDEV $\left._{t}\right) /\left(\right.$ WWBOEEXT $_{t}+$ WWBOEREV $\left._{t-1}\right)$ \\
\hline IFC3RD & $1 /\left[\left(\sum_{1=0}^{2}\right.\right.$ WWEXPL $\left._{t-1}+W_{\text {WDEV }}{ }_{t-1}\right) /\left(\sum_{1=0}^{2}\right.$ WWBOEEXT $_{t-1}+$ WWBOEREV $\left.\left._{t-1}\right)\right]$ \\
\hline \multicolumn{2}{|c|}{$\begin{array}{l}\text { Note: } \\
\text { WWEXPL is worldwide exploration expenditures. } \\
\text { WWDEV is worldwide development expenditures. } \\
\text { WWBOEEXT is worldwide reserve extensions and additions } \\
\text { measured in BOE equivalent units. } \\
\text { WWBOEREV is worldwide reserve estimate revisions measured in } \\
\text { BOE equivalent units. }\end{array}$} \\
\hline
\end{tabular}

We are interested in assessing which definition of inverse average finding costs is most strongly associated with EFF. We present two forms of analyses - one in Appendix $D$ and one in Appendix E. Appendix D reports correlation coefficients (both the Spearman rank and Pearson product moment) between EFF and the eight average inverse finding cost measures pooled across firms and across years within periods. That is, we pool inverse average finding cost measures for the years 1983-1985 for Groups 
$A$ and $B$ and compute correlation coefficients. We do the same for Groups $A$ and $C$ for the period 1990-1992. We do not compute correlations coefficients for the years 1981-1982 and 1988-1989 because computation of inverse three year moving average finding costs requires two year lags, and thus inverse moving average finding cost measures are unavailable for these years. Appendix $E$ repeats the analysis in Appendix $D$ on a year-by-year basis. We present Appendix E for completeness but largely confine our discussion to Appendix D.

The spearman rank correlations in Appendix D show that inverse average finding costs are strongly associated with EFF in both the early and late periods, and the association remains quite strong across the various definitions of inverse average finding costs. Thus, the data reject the null hypothesis that inverse average finding costs are independent of exploration efficiency as measured by EFF. However, the statistical insignificance of the Pearson correlation coefficients show that the association between EFF and inverse average finding costs is nonlinear, at least in the early period.

In both the early and later periods, inverse average finding cost measures that include exploration and development expenditures (IFC1D, IFC3D, IFCIRD, IFC3RD) consistently are more strongly associated with EFF than inverse average finding cost measures that include only exploration expenditures (IFC1, IFC3, IFCIR, IFC3R). The pattern is less consistent with respect to revision/no revisions and single-year/multiple-year measures. In 
the early period, inverse average finding cost measures that include reserve revisions (IFCIR, IFC3R, IFC1RD, IFC3RD) consistently are more strongly associated with EFF than inverse finding cost measures that exclude reserve revisions (IFC1, IFC3, IFC1D, IFC3D). In the later period, however, the presence of reserve revisions appears to interact with the time period used: Single-year inverse average finding cost measures without reserve revisions (IFC1, IFC1D) are more strongly associated with EFF than single-year finding cost measures with reserve revisions (IFClR, IFCIRD), while inverse moving-average finding costs with reserve revisions (IFC3R, IFC3RD) are more strongly associated with EFF than inverse moving-average finding costs without reserve revisions (IFC3, IFC3D). That inverse average finding cost measures which include reserve revisions are more strongly associated with EFF than those inverse average finding cost measures without revisions is anomalous if one assumes that reserve revisions represent random errors in estimating reserve quantities. One potential explanation for the result is suggested by the recent work of Clinch and Magliolo (1993) who report evidence consistent with investors discounting oil and gas stocks of firms with volatile reserve quantity estimate revisions. Their work suggests that managers bias their estimates of reserve estimates and that investors regard reserve revisions as value relevant and not as mere random estimation errors. Our findings reinforce the notion that reserve quantity revisions are more than just random estimation errors. 
The pattern of association is also less consistent with respect to the use of single-year versus moving-average inverse finding costs, with the time period and the presence of reserve revisions appearing to interact during the early period: singleyear inverse finding cost measures without reserve revisions (IFC1, IFC1D) are more strongly associated with EFF than moving average inverse finding cost measures without reserve revisions (IFC3, IFC3D), while moving-average inverse finding costs with reserve revisions (IFC3R, IFC3RD) are more strongly associated with EFF than single-year inverse finding costs with reserve revisions (IFCIR, IFCID). In the late period, three out of the four single year inverse finding costs (IFC1, IFC1R, IFCID) are more strongly associated with EFF than moving-average inverse finding costs (IFC3, IFC3R, IFC3D).

During both the early and late periods, inverse finding cost measure IFC3RD is most strongly associated with EFF, having a Spearman rank correlation coefficient of .32 and .42 in the early period and late periods, respectively. This inverse finding cost measure is a moving-average measure that includes both reserve revisions and development costs.

Additionally, a comparison of the spearman rank correlation coefficients between the two periods reveals that the strength of the association between EFF and inverse finding costs increased for all eight definitions of inverse finding costs. Apparently the usefulness of inverse finding costs as a measure of exploration efficiency has increased in recent years. 
We repeated the analysis using efficiency measures estimated from Model 2 and the results were qualitatively unchanged. We computed the Pearson product moment correlation coefficient between the EFF from model 1 and model 2 and found the two measures to be virtually the same (correlation coefficient of 0.98 significant at $\mathrm{p}=.0001$ ). Thus, our results appear robust to potential size-related omitted variables that might cause size to bias the efficiency estimates.

\section{Summary and Conclusions}

We operationalized firm-specific exploration efficiency as the difference between a firm-specific intercept estimated in a fixed-effects panel data Cobb-Douglas production frontier model and the maximum firm-specific intercept estimated in that model. The production model was estimated during two different time periods, 1982-1985 and 1989-1992, allowing efficiency to vary intertemporally. This efficiency estimate served as a benchmark against which we compared various measures of inverse finding costs. We assumed that the degree of association with an efficiency benchmark is an important attribute of any finding cost measure and that, further, the degree of association may be used as a metric for choosing between alternative finding cost measures. Accordingly, we evaluated the cross-sectional statistical association between estimated efficiency and alternative inverse finding cost measures.

We discovered that the inverse finding cost measure that exhibited the strongest association with efficiency during the 
two time periods was a three-year moving- average finding cost which included exploration plus development expenditures as costs and reserve extensions and additions plus revisions as the units added. This result is interesting for three reasons. First, our results lend empirical support for the common practice of reporting three-year or five-year moving-average finding costs in addition to single year. Second, our results lend empirical support for the argument that exploration plus development expenditures is a better measure of reserve replacement cost than exploration expenditures alone. Third, results add further documentation of an anomaly we reported a year ago in our preliminary report: Inverse finding cost measures computed with reserve revisions exhibit stronger statistical association with efficiency than those measures that exclude reserve revisions. This finding is anomalous because reserve revisions should represent random estimation errors and, therefore, would be expected to weaken the association rather than strengthen the association. That reserve revisions strengthen the association suggests that reserve revisions contain a systematic component and are not merely random estimation errors.

Finally, our study presented efficiency measures for 66 firms that survived the tumultuous decade of the 1980s, 146 firms that did not survive the decade, and 53 firms that entered the industry during the $1980 \mathrm{~s}$. The results suggest that survivor firms were more efficient than non-survivors or new firms, and that non-survivor firms were least efficient among the three 
groups. The results also show that the relative dispersion in efficiency measures decreased during the period 1989-1992 as compared to the relative dispersion during the period 1982-1985, suggesting that industry restructuring produced a more homogeneous set of firms.

\section{Further studies}

This study suggests two potentially fruitful avenues for future work. First, the finding that inverse finding cost measures are associated with exploration efficiency implies that finding cost measures may provide useful information to investors if knowledge of relative exploration efficiency assists investors in assessing a firm's future cash flows or systematic risk. Understanding whether investors use finding costs in pricing oil and gas common stocks would provide insight into the actual relevance of finding cost measures. Thus, one avenue for future research would be to test whether finding cost measures convey useful information to investors.

Second, the finding that reserve revisions may contain a systematic component begs the question of what that component might be. Clinch and Magliolo (1992) similarly conclude that reserve revisions appear to contain systematic aspects, and they find this result puzzling because they see no apparent reason why management would benefit from biasing their reserve estimates. Future research could attempt to identify and explain the apparent systematic component of reserve revisions. 
APPENDIX A

PRODUCTION MODELS 
Appendix $A$

1982-1985 Time Period

Group A (Survivors) and Group B (Non-survivors)

(Printing of firm Dummies suppressed)

Model: Model 1

Dependent Variable: LNADD
09:38 Sunday September 25, 1994

Analysis of Variance

\begin{tabular}{|c|c|c|c|c|c|}
\hline Source & $D F$ & $\begin{array}{l}\text { Sum of } \\
\text { Squares }\end{array}$ & $\begin{array}{r}\text { Mean } \\
\text { Square }\end{array}$ & F Value & Prob $>F$ \\
\hline $\begin{array}{l}\text { Model } \\
\text { Error } \\
\text { C Total }\end{array}$ & $\begin{array}{l}216 \\
631 \\
847\end{array}$ & $\begin{array}{r}4033.85682 \\
448.53700 \\
4482.39382\end{array}$ & $\begin{array}{r}18.67526 \\
0.71084\end{array}$ & 26.272 & 0.0001 \\
\hline $\begin{array}{l}\text { Root MSE } \\
\text { Dep Mean } \\
\text { C.V. }\end{array}$ & & $\begin{array}{l}0.84311 \\
7.21897 \\
1.67909\end{array}$ & $\begin{array}{l}R \text {-square } \\
\text { Adj } R \text {-sq }\end{array}$ & $\begin{array}{l}0.8999 \\
0.8657\end{array}$ & \\
\hline
\end{tabular}

Parameter Estimates

$\begin{array}{lcrrrrl}\text { Variable } & \text { DF } & \begin{array}{r}\text { Parameter } \\ \text { Estimate }\end{array} & \begin{array}{r}\text { Standard } \\ \text { Error }\end{array} & \begin{array}{r}\text { T for HO: } \\ \text { Parameter=0 }\end{array} & \text { Prob > }|T| \begin{array}{r}\text { Variable } \\ \text { Label }\end{array} \\ \text { INTERCEP } & 1 & 1.041721 & 0.66102802 & 1.576 & 0.1155 & \text { Intercept } \\ \text { LNEXP } & 1 & 0.186407 & 0.04809661 & 3.876 & 0.0001 & \text { LOG EXPLORATION COSTS } \\ \text { LNDEV } & 1 & 0.343057 & \mathbf{0 . 0 5 2 3 4 7 5 4} & 6.553 & 0.0001 & \text { LOG DEVELOPMENT COSTS } \\ \text { LNOUT } & 1 & -0.041525 & 0.08678492 & -0.478 & 0.6325 & \text { LOG PROOUCTION IN BOE } \\ \text { LAGEXP } & 1 & -0.020541 & 0.04841353 & -0.424 & 0.6715 & \text { LAG LNEXP } \\ \text { LAGDEV } & 1 & 0.027625 & \mathbf{0 . 0 4 7 6 8 2 8 9} & 0.579 & 0.5626 & \text { LAG LNDEV }\end{array}$


Production Model for 1982-1985 Time Period Group A (Survivors) and Group B (Non-survivors) Test of Linear Restrictions

Dependent Variable: LNADD Test: R1

Dependent Variable: LNADD Test: R2

Dependent Variable: LNADO Test: R3

Dependent Variable: LNADO Test: $\mathbf{R 4}$

19.7045 DF:

0.710835 DF:

merator:

19.6086

Denominator

0.710835

DF: 1

DF: 631

F value:

Prob>F:

0.0001

\begin{tabular}{|c|c|c|c|c|c|}
\hline $\begin{array}{l}\text { Numerator: } \\
\text { Denominator: }\end{array}$ & $\begin{array}{r}19.5218 \\
0.710835\end{array}$ & $\begin{array}{l}\text { DF: } \\
\text { DF: }\end{array}$ & $\begin{array}{r}1 \\
631\end{array}$ & $\begin{array}{l}\text { F value: } \\
\text { Prob>F: }\end{array}$ & $\begin{array}{r}27.4632 \\
0.0001\end{array}$ \\
\hline $\begin{array}{l}\text { Numerator: } \\
\text { Denominator: }\end{array}$ & $\begin{array}{r}0.1510 \\
0.710835\end{array}$ & $\begin{array}{l}\text { DF: } \\
\text { DF: }\end{array}$ & $\begin{array}{r}2 \\
631\end{array}$ & $\begin{array}{l}\text { F value: } \\
\text { Prob>F: }\end{array}$ & $\begin{array}{l}0.2124 \\
0.8087\end{array}$ \\
\hline
\end{tabular}

09:38 Sunday September 25, 1994

R1: LNEXP + LNOEV+LNOUT $=1$

R2: $L N E X P+L N O E V+L N O U T+L A G E X P=1$

R3: LNEXP+LNDEV+LNOUT+LAGEXP+LAGDEV $=1$

R4: $L A G E X P=\angle A G D E V=0$ 
Appendix A

Production Model for 1989-1992 Time Period Group A (Survivors) and Group C (New Entrants)

(Printing of Firm Dumnies Suppressed)

Model: Model 1

Dependent Variable: LNADD
09:38 Sunday September 25, 1994

\section{Analysis of Variance}

\begin{tabular}{|c|c|c|c|c|c|}
\hline Source & $D F$ & $\begin{array}{l}\text { Sum of } \\
\text { squares }\end{array}$ & $\begin{array}{r}\text { Mean } \\
\text { Square }\end{array}$ & F Value & Prob $>F$ \\
\hline $\begin{array}{l}\text { Model } \\
\text { Error }\end{array}$ & $\begin{array}{l}123 \\
352\end{array}$ & $\begin{array}{r}2280.20305 \\
258.78304\end{array}$ & $\begin{array}{r}18.53824 \\
0.73518\end{array}$ & 25.216 & 0.0001 \\
\hline
\end{tabular}

Error

$\begin{array}{lr}352 & 258.78304\end{array}$

0.73518

Root MSE

Dep Mean

$475 \quad 2538.98609$

C.v.
0.85743
R-square
0.8981
8.42914 Adj R-sq 0.8625

10.17217

Parameter Estimates

$\begin{array}{lcrrrrl}\text { Variable } & \text { DF } & \begin{array}{r}\text { Parameter } \\ \text { Estimate }\end{array} & \begin{array}{r}\text { Standard } \\ \text { Error }\end{array} & \begin{array}{r}\text { T for HO: } \\ \text { Parameter=0 }\end{array} & \text { Prob > }|\mathrm{T}| \begin{array}{r}\text { Variable } \\ \text { Label }\end{array} \\ \text { INTERCEP } & 1 & 0.830332 & 1.01387661 & 0.819 & 0.4134 & \text { Intercept } \\ \text { LNEXP } & 1 & 0.333934 & 0.06687624 & 4.993 & 0.0001 & \text { LOG EXPLORATION COSTS } \\ \text { LNDEV } & 1 & 0.501753 & 0.09017741 & 5.564 & 0.0001 & \text { LOG DEVELOPMENT COSTS } \\ \text { LNOUT } & 1 & -0.069418 & 0.25254020 & -0.275 & 0.7836 & \text { LOG PROUCTION IN BOE } \\ \text { LAGEXP } & 1 & -0.048903 & 0.07280234 & -0.672 & 0.5022 & \text { LAG LNEXP } \\ \text { LAGDEV } & 1 & -0.006336 & 0.08773834 & -0.072 & 0.9425 & \text { LAG LNDEV }\end{array}$


Appendix A

Production Model for 1989-1992 Time Period Group A (Survivors) and Group C (New Entrants) Test of Linear Restrictions

Dependent Variable: LNADD

Test: R1

Dependent Variable: LNADD Test: R2

Dependent Variable: LNADD

Test: R3

Dependent Variable: LNADD Test: $R 4$

Numerator:

0.6616 DF: 1 F value

value:

0.9000

Denominator:

0.735179 DF:

Numerator:

1.0662 DF:

F value:

0.735179 DF: 352 Prob>F: 0.2293

Numerator:

1.5153 DF: 1

Denominator:

1.5153
0.735179

DF: 35

F value:

2.0612

Numerator:

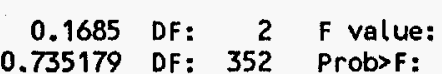

0.1520

Denominator:

Prob>F :

0.2292

0.7953

R1: LNEXP +LNDEV+LNOUT $=1$

R2: $L N E X P+L N D E V+L N O U T+\angle A G E X P=1$

R3: LNEXP+LNDEV+LNOUT+LAGEXP+LAGDEV $=1$

R4: LAGEXP $=\angle A G D E V=0$
09:38 Sunday September 25, 1994 
Appendix $A$

Model for 1982-1985 Time Period

Group A (Survivors) and Group B (Non-survivors)

(Printing of Firm Dummies suppressed)

Model: MOOEL2

Dependent Variable: LNADD
Analysis of Variance

\begin{tabular}{lrrrrr} 
Source & DF & $\begin{array}{r}\text { Sum of } \\
\text { Squares }\end{array}$ & $\begin{array}{r}\text { Mean } \\
\text { Square }\end{array}$ & F Value & Prob>F \\
Model & 217 & 4037.62804 & 18.60658 & 26.356 & 0.0001 \\
Error & 630 & 444.76578 & 0.70598 & & \\
C Total & 847 & 4482.39382 & & & \\
\multicolumn{7}{c}{} & & & \\
$\quad$ Root MSE & 0.84022 & R-square & 0.9008 & \\
$\quad$ Dep Mean & 7.21897 & Adj R-sq & 0.8666 & \\
C.V. & 11.63911 & & & &
\end{tabular}

Parameter Estimates

\begin{tabular}{|c|c|c|c|c|c|c|}
\hline Variable & DF & $\begin{array}{r}\text { Parameter } \\
\text { Est imate }\end{array}$ & $\begin{array}{r}\text { Standard } \\
\text { Error }\end{array}$ & $\begin{array}{c}T \text { for } \mathrm{HO}^{\circ} \\
\text { Parameter=0 }\end{array}$ & Prob $>|T|$ & $\begin{array}{c}\text { Variable } \\
\text { Label }\end{array}$ \\
\hline $\begin{array}{l}\text { INTERCEP } \\
\text { LNEXP } \\
\text { LNDEV } \\
\text { LNOUT } \\
\text { LAGEXP } \\
\text { LAGDEV } \\
\text { LNTRB }\end{array}$ & $\begin{array}{l}1 \\
1 \\
1 \\
1 \\
1 \\
1 \\
1\end{array}$ & $\begin{array}{r}1.884333 \\
0.177908 \\
0.342229 \\
0.144331 \\
-0.012977 \\
0.040856 \\
-0.272307\end{array}$ & $\begin{array}{l}0.75291702 \\
0.04807283 \\
0.05216959 \\
0.11809530 \\
0.04835870 \\
0.04786325 \\
0.11781855\end{array}$ & $\begin{array}{r}2.503 \\
3.701 \\
6.560 \\
1.222 \\
-0.268 \\
0.854 \\
-2.311\end{array}$ & $\begin{array}{l}0.0126 \\
0.0002 \\
0.0001 \\
0.2221 \\
0.7885 \\
0.3937 \\
0.0211\end{array}$ & $\begin{array}{l}\text { Intercept } \\
\text { LOG EXPLORATION COSTS } \\
\text { LOG DEVELOPMENT COSTS } \\
\text { LOG PROOUCTION IN BOE }\end{array}$ \\
\hline
\end{tabular}

LNTRB

0.0211

BEGIN. BOE RESERVES
09:38 Sunday September 25, 1994 
Appendix A

Production Model for 1982-1985 Time Period Group A (Survivors) and Group B (Non-survivors) Test of Linear Restrictions

Dependent Variable: LNADD Test: R1

Dependent Variable: LNADD Test: R2

Dependent Variable: LNADD Test: R3

Dependent Variable: LNADO Test: R4

R1: LNEXP+LNDEV+LNOUT+LNTRB $=1$

R2: $L N E X P+L N D E V+L N O U T+L A G E X P=1$

R3: LNEXP+LNDEV+LNOUT+LAGEXP+LAGDEV $=1$

R4: LAGEXP $=\angle A G D E V=0$
Numerator:
23.4740 DF: 1
value: 33.2504
Denominator:
0.705977 DF: 630
Prob>F :
0.0001
Numerator:
5.1788 OF:
F value:
7.3357
Denominator:
0.705977 DF: 630
Prob>F :
0.0069
Numerator:
4.0471 DF: 1 F value: 5.7326
Denominator: 0.705977 DF: 630 Prob>F: 0.0169

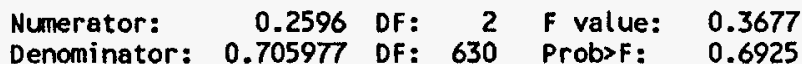

09:38 Sunday September 25, 1994 
Appendix A

Production Model for 1989-1992 Time Period Group A (Survivors) and Group $C$ (New Entrants)

(Printing of Firm Dumies Suppressed)

Model: Model2

Dependent Variable: LNADD
Analysis of Variance

$\begin{array}{lrrrrr}\text { Source } & \text { DF } & \begin{array}{r}\text { Sum of } \\ \text { Squares }\end{array} & \begin{array}{r}\text { Mean } \\ \text { Square }\end{array} & \text { F Value } & \text { Prob>F } \\ \text { Model } & 124 & 2280.86814 & 18.39410 & 25.013 & 0.0001 \\ \text { Error } & 351 & 258.11795 & 0.73538 & & \end{array}$

Error

$\begin{array}{lr}375 & 258.11795 \\ & 2538.98609\end{array}$

Root MSE

Dep Mean

Dep Mea
C.V.

0.85754
8.42914
10.17355

$R$-square

0.8983

Adj $R$-sq

0.8624

Parameter Estimates

$\begin{array}{lcrrrrl}\text { Variable } & \text { DF } & \begin{array}{r}\text { Parameter } \\ \text { Estimate }\end{array} & \begin{array}{r}\text { Standard } \\ \text { Error }\end{array} & \begin{array}{r}\text { T for HO: } \\ \text { Parameter }=0\end{array} & \text { Prob > |T| } \begin{array}{c}\text { Variable } \\ \text { Label }\end{array} \\ \text { INTERCEP } & 1 & 0.495420 & 1.07342652 & 0.462 & 0.6447 & \text { Intercept } \\ \text { LNEXP } & 1 & 0.333010 & 0.06689237 & 4.978 & 0.0001 & \text { LOG EXPLORATION COSTS } \\ \text { LNDEV } & 1 & 0.492210 & 0.09074621 & 5.424 & 0.0001 & \text { LOG DEVELOPMENT COSTS } \\ \text { LNOUT } & 1 & -0.179937 & 0.27802736 & -0.647 & 0.5179 & \text { LOG PRODUCTION IN BOE } \\ \text { LAGEXP } & 1 & -0.051512 & 0.07286392 & -0.707 & 0.4801 & \\ \text { LAGDEV } & 1 & -0.039133 & 0.09428379 & -0.415 & 0.6784 & \\ \text { LNTRB } & 1 & 0.156919 & 0.16500215 & 0.951 & 0.3423 & \text { LOG BEGIN. BOE RESERVES }\end{array}$

0.156919

0.16500215

0.3423

LO BEGIN. BOE RESERVES
09:38 Sunday September 25, 1994 
Dependent Variable: LNADD Test: R1

Dependent Variable: LNADD

Test: R2

Dependent Variable: LNADD Test: R3

Dependent Variable: LNADD Test: $R 4$

R1: $L N E X P+L N D E V+L N O U T+L N T R B=1$

R2: $L N E X P+L N D E V+L N O U T+L A G E X P=1$

R3: $L N E X P+L N D E V+L N O U T+L A G E X P+L A G D E V=1$

R4: $L A G E X P=L A G D E V=0$
Appendix A

Production Model for 1989-1992 Time Period Group A (Survivors) and Group C (New Entrants) Test of Linear Restrictions

09:38 sunday September 25, 1994

Numerator: $\quad 0.4630$ DF: 1 F value: 0.6296

Denominator: 0.735379 DF: 351 Prob>F: 0.4281

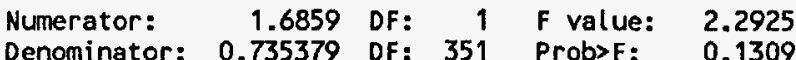

Numerator: 2.1588 OF : 1 F value: 2.9356

Denominator: 0.735379 of: 351 Prob>F: 0.087

Numerator: $\quad 0.2480$ DF: 2 F value: 0.3372

Denominator: 0.735379 DF: 351 ProbsF: 0.7140 


\section{APPENDIX B}

\section{FIRM SPECIFIC EFFICIENCY MEASURES}


Appendix B

For Group A (Survivors) and Group B (Non-survivors) - 1982-1985

\begin{tabular}{|c|c|c|c|c|c|}
\hline OBS & NAME & SMBL & EFF & & GROUP \\
\hline 1 & EXXON & XON & 1.00000 & A - & survivors \\
\hline 2 & TENNECO & TGT & 0.60001 & B & Non-survi \\
\hline 3 & SHELL OIL & $4108 \mathrm{~A}$ & 0.54318 & B & Non-survi \\
\hline 4 & ATLANTIC RICHFIELD (ARCO) & ARC & 0.51767 & A & Survivors \\
\hline 5 & ** TEXAS OIL \& GAS & TXO & 0.45955 & B - & Non-survi \\
\hline 6 & UNOCAL (UNION OIL OF CA) & UCL & 0.45250 & A - & Survivors \\
\hline 7 & SUN COMPANY & SUN & 0.40726 & A & Survivors \\
\hline 8 & AMOCO (STD OF INDIANA) & AN & 0.38217 & & Survivors \\
\hline 9 & MOBIL & MOB & 0.35055 & A - & Survivors \\
\hline 10 & OCCIDENTAL PETRO. & OXY & 0.34969 & A & Survivors \\
\hline 11 & DUPONT (CONOCO) & DD & 0.33724 & A & Survivors \\
\hline 12 & CHEVRON (STD CALIFORNIA) & CHV & 0.31772 & A - & Survivors \\
\hline 13 & UNION PACIFIC & UNP & 0.31235 & A & Survivors \\
\hline 14 & CANADIAN PACIFIC LTD. & $C P$ & 0.29291 & A - & Survivors \\
\hline 15 & TEXACO & $\mathrm{TX}$ & 0.27611 & A & Survivors \\
\hline 16 & ENSERCH & ENS & 0.25191 & & Survivors \\
\hline 17 & DOME PETROLEUM & DMP & 0.24107 & B - & Non-survi \\
\hline 18 & CONSOL. NAT. GAS & CNG & 0.23621 & A & survivors \\
\hline 19 & TEXAS EASTERN CORP. & TET & 0.22930 & B & Non-survi \\
\hline 20 & PANHANOLE EASTERN & PEL & 0.18882 & B & Non-survi \\
\hline 21 & MITCHELL ENERGY & MND & 0.18637 & $A$ & Survivors \\
\hline 22 & ** PETRO-LEWIS & 4356B & 0.18603 & B & Non-survi \\
\hline 23 & & $P$ & $\begin{array}{l}0.18352 \\
0.18251\end{array}$ & $\begin{array}{l}\text { A } \\
\text { B }\end{array}$ & $\begin{array}{l}\text { Survivors } \\
\text { Non-survi }\end{array}$ \\
\hline $\begin{array}{l}24 \\
25\end{array}$ & $\begin{array}{l}\text { DIAROND SHAMROCK } \\
\text { KERR-MCGEE CORPORATION }\end{array}$ & KMG & 0.18079 & A & Survivors \\
\hline 26 & NOBLE AFFILIATES & NBL & 0.17622 & A & Survivors \\
\hline 27 & MURPHY OIL & MUR & 0.17055 & A & Survivors \\
\hline 28 & PENNZOIL & PZL & 0.16833 & A & Survivors \\
\hline 29 & MONOGRAM OIL \& GAS & $5235 B$ & 0.16288 & B & Non-survi \\
\hline 30 & CABOT CORP. & CBT & 0.15784 & A & Survivors \\
\hline 31 & ENRON (HNG/INTERNORTH) & ENE & 0.15698 & B & Non-survi \\
\hline 32 & ARKLA & ALG & 0.15471 & B & Non-survi \\
\hline 33 & DEKALB CORP & ENRGB & 0.14934 & A & Survivors \\
\hline 34 & ** HIRAM WALKER RES & D.HAV & 0.14849 & B & Non-survi \\
\hline 35 & $\begin{array}{l}\text { ODECO } \\
\text { ARGO PETROLEUM }\end{array}$ & $\begin{array}{l}\text { ODR } \\
4268 B\end{array}$ & $\begin{array}{l}0.14369 \\
0.13673\end{array}$ & $\begin{array}{l}\text { B } \\
\text { B }\end{array}$ & $\begin{array}{l}\text { Non-survi } \\
\text { Non-survi }\end{array}$ \\
\hline 37 & $\begin{array}{l}\text { ARGO PETROLEUM } \\
\text { ASAMERA }\end{array}$ & ASM & 0.13652 & B & Non-survi \\
\hline 38 & $\begin{array}{l}\text { ASAMERA } \\
\text { WORLDWIDE ENERGY }\end{array}$ & WWE & 0.13584 & B & Non-survi \\
\hline 39 & ENERGY OIL & EOI & 0.13470 & B & Non-survi \\
\hline 40 & EQUITABLE RESOURCES & EQT & 0.13439 & A & Survivors \\
\hline
\end{tabular}


Appendix $B$

09:38 Sunday September 25, 1994

Firm-Specific Efficiency Measures

For Group A (Survivors) and Group B (Non-survivors) - 1982-1985

\begin{tabular}{|c|c|c|c|c|c|}
\hline OBS & NAME & SMBL & EFF & & GROUP \\
\hline $\begin{array}{l}41 \\
42 \\
43 \\
44 \\
45 \\
46 \\
47 \\
48 \\
49 \\
50 \\
51 \\
52 \\
53 \\
54 \\
55 \\
56 \\
57 \\
58 \\
59 \\
60 \\
61 \\
62 \\
63 \\
64 \\
65 \\
66 \\
67 \\
68 \\
69 \\
70 \\
71 \\
72 \\
73 \\
74 \\
75 \\
76 \\
77 \\
78 \\
79 \\
80\end{array}$ & $\begin{array}{l}\text { SANTA FE SO. PACIFIC } \\
\text { AMERADA HESS } \\
\text { COASTAL } \\
\text { CRYSTAL OIL } \\
\text { ** INEXCO OIL } \\
\text { AMERICAN PETROFINA } \\
\text { READING \& BATES } \\
\text { HUSKY OIL } \\
\text { HAMILTON OIL CORP } \\
\text { TRANSCO ENERGY } \\
\text { STANDARD OIL (SOHIO) } \\
\text { LOUISIANA LAND \& EXPL. } \\
\text { WESTCOAST TRANSMISSION } \\
\text { HOWELL CORP. } \\
\text { MESA LTD. P SHIP (MLP) } \\
\text { APACHE CORP. } \\
\text { CONQUEST EXPL. } \\
\text { TEXAS GAS RES. (CSX) } \\
\text { GOODYEAR/CELERON } \\
\text { LEAR PET. PTRS. (MLP) } \\
\text { TOTAL PETROLEUM } \\
\text { BOW VALLEY IND. } \\
\text { NUMAC OIL \& GAS LTD } \\
\text { FREEPORT-MCMORAN } \\
\text { TEXAS INT L } \\
\text { ASHLAND OIL } \\
\text { POGO PROOUCING CO. } \\
\text { ** UNIVERSAL RESOURCES } \\
\text { ** MIDCON } \\
\text { SOUTHERN UNION COMPANY } \\
\text { PACIFIC LIGHTING CORP. } \\
\text { SOUTHDOWN INC } \\
\text { CONSOL. OIL \& GAS } \\
\text { PARK-OHIO INDUS. } \\
\text { SCURRY RAINBOW OIL } \\
\text { SABINE CORP. } \\
\text { INTER-CITY GAS } \\
\text { QUESTAR CORPORATION } \\
\text { COLUMBIA GAS SYS. } \\
\text { NICOR }\end{array}$ & $\begin{array}{l}\text { SFX } \\
\text { AHC } \\
\text { CGP } \\
\text { COR } \\
\text { INX } \\
\text { API.A } \\
\text { RB } \\
\text { HYO } \\
\text { HAML } \\
\text { E } \\
\text { SRD } \\
\text { LLX } \\
\text { WTC } \\
\text { HWL } \\
\text { MLP } \\
\text { APA } \\
\text { CQX } \\
\text { CSX } \\
\text { GT } \\
\text { LPP } \\
\text { TPN } \\
\text { BVI } \\
\text { NMC } \\
\text { FTX } \\
\text { TEICC } \\
\text { ASH } \\
\text { PPP } \\
\text { UVR } \\
\text { MCN.1 } \\
\text { SUG } \\
\text { PLT } \\
\text { SOW } \\
\text { CGS } \\
\text { PKOH } \\
\text { SRB } \\
\text { SAB } \\
\text { ICG } \\
\text { STR } \\
\text { CG } \\
\text { GAS }\end{array}$ & $\begin{array}{l}0.13380 \\
0.13327 \\
0.13218 \\
0.13033 \\
0.12980 \\
0.12473 \\
0.12461 \\
0.12439 \\
0.12437 \\
0.12406 \\
0.12314 \\
0.11866 \\
0.11778 \\
0.11749 \\
0.11737 \\
0.11553 \\
0.11314 \\
0.11245 \\
0.11024 \\
0.10827 \\
0.10701 \\
0.10696 \\
0.10485 \\
0.10443 \\
0.09936 \\
0.09532 \\
0.09468 \\
0.09362 \\
0.09267 \\
0.09251 \\
0.08819 \\
0.08818 \\
0.08801 \\
0.08791 \\
0.08671 \\
0.08620 \\
0.08541 \\
0.08471 \\
0.08369 \\
0.08221\end{array}$ & $\begin{array}{l}B= \\
A= \\
A= \\
A= \\
B= \\
B= \\
B= \\
B= \\
B= \\
B= \\
B= \\
A= \\
B= \\
A= \\
B= \\
A= \\
B= \\
B= \\
B= \\
B= \\
B= \\
A= \\
A= \\
B= \\
B= \\
A= \\
A= \\
B= \\
B= \\
B= \\
B= \\
B= \\
B= \\
B= \\
B= \\
B= \\
B= \\
A= \\
A= \\
A=\end{array}$ & $\begin{array}{l}\text { Non-survi } \\
\text { Survivors } \\
\text { Survivors } \\
\text { Survivors } \\
\text { Non-survi } \\
\text { Non-survi } \\
\text { Non-survi } \\
\text { Non-survi } \\
\text { Non-survi } \\
\text { Non-survi } \\
\text { Non-survi } \\
\text { Survivors } \\
\text { Non-survi } \\
\text { Survivors } \\
\text { Non-survi } \\
\text { Survivors } \\
\text { Non-survi } \\
\text { Non-survi } \\
\text { Non-survi } \\
\text { Non-survi } \\
\text { Non-survi } \\
\text { Survivors } \\
\text { Survivors } \\
\text { Non-survi } \\
\text { Non-survi } \\
\text { Survivors } \\
\text { Survivors } \\
\text { Non-survi } \\
\text { Non-survi } \\
\text { Non-survi } \\
\text { Non-survi } \\
\text { Non-survi } \\
\text { Non-survi } \\
\text { Non-survi } \\
\text { Non-survi } \\
\text { Non-survi } \\
\text { Non-survi } \\
\text { Survivors } \\
\text { Survivors } \\
\text { Survivors }\end{array}$ \\
\hline
\end{tabular}


Appendix $B$

Firm-Specific Efficiency Measures

09:38 Sunday September 25,1994

For Group A (Survivors) and Group B (Non-survivors) - 1982-1985

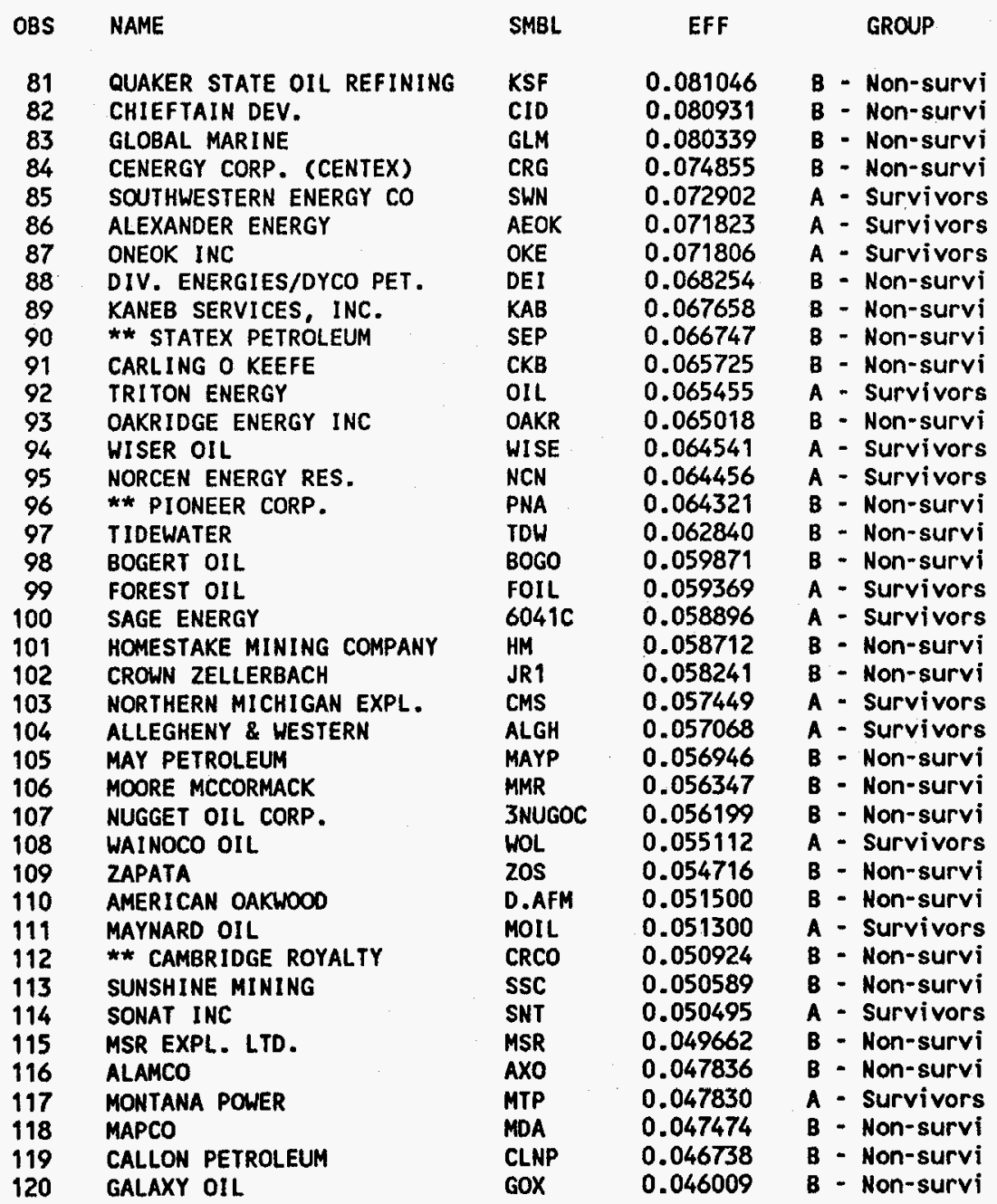


Appendix B

For Group A (Survivors) and Group B (Non-survivors) - 1982-1985

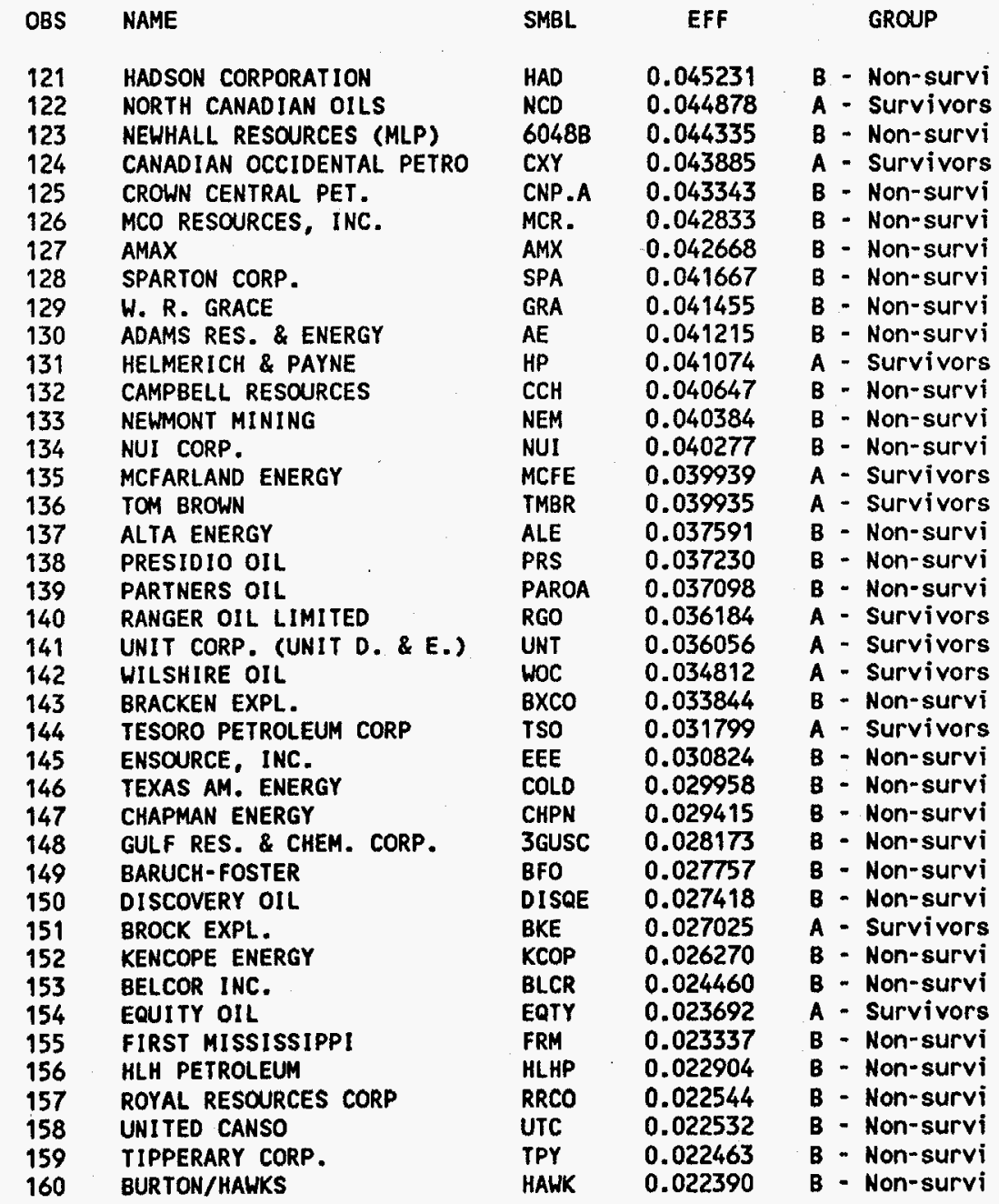


Appendix B

Firm-Specific Efficiency Measures

For Group A (Survivors) and Group B (Non-survivors) - 1982-1985

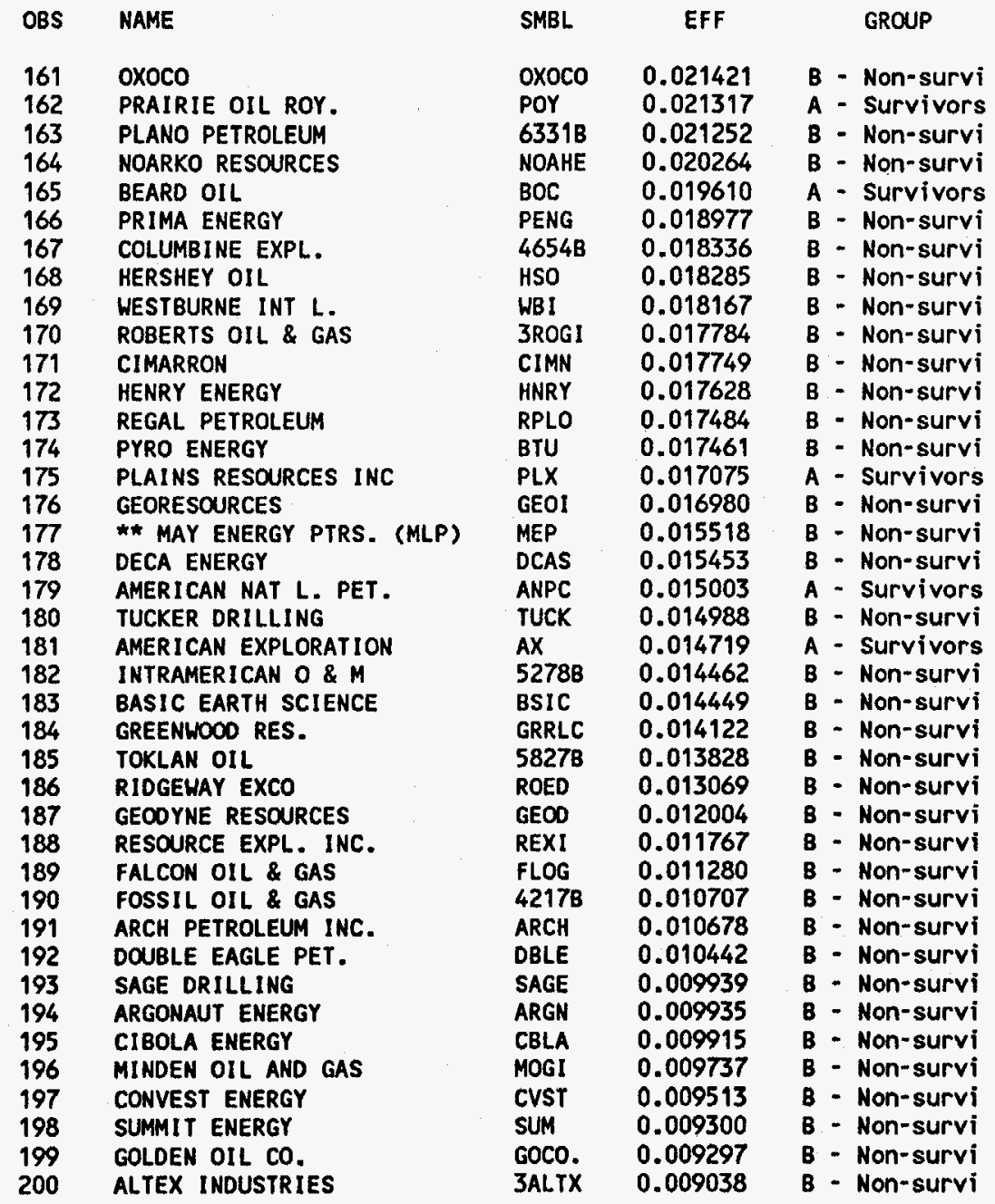


Appendix $B$

Firm-Specific Efficiency Measures

For Group A (Survivors) and Group B (Non-survivors) - 1982-1985

OBS NAME

ASPEN EXPL.

GREAT EAST. ENERGY WICHITA INDUSTRIES

PAULEY PETROLEUM

ARAPAHO PETROLEUM

MARLIN OIL

PANGEA PETROLEUM

PARALLEL PETROLEUM

KRM PETROLEUM CORPORATION

KIRBY EXPLORATION CO INC

SO. TEXAS DRLG. \& EX.

TOMLINSON OIL
SMBL

3AXPN

GREN

WRO

PP

ARHO

MRLNE

PPET

PLLL

KRMC

KEX

3STXD

$4566 \mathrm{~B}$
EFF

GROUP

.0078642

.0075614

.0070882

.0068581

0066660

.0056509

.0049875

.0041939

.0040455

.0023458 
Appendix B

Firm-Specific Efficiency Measures

For Group A (Survivors) and Group C (New Entrants) - 1989-1992

OBS NAME

UNOCAL CORP

BURLINGTON RESOURCES INC

ROYAL DUTCH/SHELL GRP COMB

ORYX ENERGY CO

SOCIETE NATIONALE EL.F AQUITAINE

PHILLIPS PETROLEUM CO

ATLANTIC RICHFIELD CO

BRITISH PETROLEUM PLC -ADR

CABOT CORP

MITCHELL ENERGY \& DEV

ENRON OIL \& GAS CO.

THE PHOENIX RESOURCE COMPANIES, INC.

UNION PACIFIC CORP

SOUTHWESTERN ENERGY CO

USX CORPORATION

EXXON CORP

MAXUS ENERGY CORP

TEXACO INC

WASHINGTON ENERGY CO

AMOCO CORP

CMS ENERGY CORP

KERR-MCGEE CORP

BHP

MOBIL CORP

CONSOLIDATED NATURAL GAS CO

PRESIDIO OIL COMPANY

SAGE ENERGY CO.

TOTAL.

TRITON ENERGY CORP

NORCEN ENERGY RES

NORTH CANADIAN OILS LTO

HALL WOOD ENERGY CORPORATION

BARRETT RESOURCES CORP

APACHE CORP

CHEVRON CORP

ENERGEN CORP

COASTAL CORP

CANADIAN OCCIDENTAL PETRO

BROWN (TOM) INC

OCCIDENTAL PETROLEUM CORP

$\begin{array}{lcc}\text { SMBL } & \text { EFF } & \text { GROUP } \\ \text { UCL } & 1.00000 & \text { A - Survivors } \\ \text { BR } & 0.98368 & \text { C - New Entra } \\ \text { RDSC.CM } & 0.79527 & \text { C - NeW Entra } \\ \text { ORX } & 0.69887 & \text { C - New Entra } \\ \text { ELF } & 0.66998 & \text { C - New Entra } \\ \text { P } & 0.66496 & \text { A - Survivors } \\ \text { ARC } & 0.61510 & \text { A - Survivors } \\ \text { BP } & 0.60761 & \text { C - New Entra } \\ \text { CBT } & 0.57554 & \text { A - Survivors } \\ \text { MND } & 0.49312 & \text { A - Survivors } \\ \text { EOG } & 0.48677 & \text { C - New Entra } \\ \text { PHNI } & 0.48501 & \text { C - New Entra } \\ \text { UNP } & 0.47922 & \text { A - Survivors } \\ \text { SWN } & 0.47453 & \text { A - Survivors } \\ \text { MROX.CM } & 0.47282 & \text { C - New Entra } \\ \text { XON } & 0.46386 & \text { A - Survivors } \\ \text { MXS } & 0.43804 & \text { C - New Entra } \\ \text { TX } & 0.43759 & \text { A - Survivors } \\ \text { WEG } & 0.43571 & \text { C - New Entra } \\ \text { AN } & 0.42980 & \text { A - Survivors } \\ \text { CMS } & 0.41521 & \text { A - Survivors } \\ \text { KMG } & 0.41276 & \text { A - Survivors } \\ \text { BHP } & 0.39783 & \text { C - New Entra } \\ \text { MOB } & 0.39020 & \text { A - Survivors } \\ \text { CNG } & 0.38499 & \text { A - Survivors } \\ \text { PRS.B } & 0.37253 & \text { C - New Entra } \\ \text { GO41C } & 0.36118 & \text { A - Survivors } \\ \text { TOT } & 0.35472 & \text { C - New Entra } \\ \text { OIL } & 0.35046 & \text { A - Survivors } \\ \text { NCN } & 0.34286 & \text { A - Survivors } \\ \text { NCD } & 0.34146 & \text { A - Survivors } \\ \text { HEP } & 0.34041 & \text { C - New Entra } \\ \text { BARC } & 0.33418 & \text { C - New Entra } \\ \text { APA } & 0.33102 & \text { A - Survivors } \\ \text { CHV } & 0.32801 & \text { A - Survivors } \\ \text { EGN } & 0.32668 & \text { C - New Entra } \\ \text { CGP } & 0.32496 & \text { A - Survivors } \\ \text { CXY } & 0.32410 & \text { A - Survivors } \\ \text { TMBR } & 0.32331 & \text { A - Survivors } \\ \text { OXY } & 0.32213 & \text { A - Survivors } \\ & & \end{array}$


Appendix B

Firm-Specific Efficiency Measures

For Group A (Survivors) and Group C (New Entrants) - 1989-1992

OBS NAME

COLUMBIA GAS SYSTEM

DU PONT (E.I) DE NEMOURS

NOBLE AFFILIATES INC

ALEXANDER ENERGY CORP

ANADARKO PETROLEUM CORP

TESORO PETROLEUM CORP

QUESTAR CORP

MURPHY OIL CORP

CANADIAN PACIFIC LIMITED

BOW VALLEY INDUSTRIES LTD

SONAT INC

PENNZOIL CO

AMERICAN NATIONAL PETROLEUM

POGO PROOUCING CO

ENSERCH CORP

GARNET RESOURCES CORP

HOME OIL COMPANY LTD.

ASHLAND OIL INC

NATIONAL FUEL GAS CO

FINA, INC.

NICOR INC

LOUISIANA LAND \& EXPLORATION

MESA INC.

EQUITABLE RESOURCES INC

MONTANA POWER CO

SCEPTRE RESOURCES LTD

EDISTO RESOURCES CP

NAHAMA \& WEAGANT ENERGY COMPANY

KCS ENERGY, INC.

HELMERICH \& PAYNE

AMERADA HESS CORP

BRITISH GAS

WAINOCO OIL CORP

GULF CANADA RES LTD -ORD

WISER OIL CO

DEKALB ENERGY CO -CL B

PRAIRIE OIL ROYALTIES CO LTO

NEH LONDON INC

SEAGULL ENERGY CORP

UNIT CORP
SMBL

EFF

GROUP

\begin{tabular}{|c|c|c|c|}
\hline $\mathrm{CG}$ & 0.31842 & A & survivors \\
\hline DD & 0.31652 & A & - survivors \\
\hline NBL & 0.31423 & A & Survivors \\
\hline & & A & Survivors \\
\hline APC & 0.29820 & C & - New Entra \\
\hline TSO & 0.29407 & & - survivors \\
\hline STR & 0.29286 & A & - Survivors \\
\hline MUR & 0.29151 & A & Survivors \\
\hline$C P$ & 0.28638 & A & Survivors \\
\hline BVI & 0.28323 & A & Survivors \\
\hline SNT & 0.28066 & A & - survivors \\
\hline PZL & 0.27993 & A & - survivors \\
\hline ANPC & 0.27270 & A & - Survivors \\
\hline PPP & 0.27108 & A & Survivors \\
\hline ENS & 0.27082 & A & - survivors \\
\hline GARN & 0.26900 & C & - New Entra \\
\hline HO & 0.26870 & C & New Entra \\
\hline ASH & 0.26443 & A & urvivors \\
\hline NFG & 0.25426 & C & New Entra \\
\hline FI & 0.25100 & C & New Entra \\
\hline GAS & 0.24200 & A & Survivors \\
\hline LLX & 0.24110 & A & - Survivors \\
\hline MXP & 0.24016 & C & New Entra \\
\hline EQT & 0.23963 & A & urvivors \\
\hline MTP & 0.23155 & A & Survivors \\
\hline SRL & 0.22436 & C & New Entra \\
\hline EDS & 0.22311 & C & New Entra \\
\hline NAWE & 0.21735 & c & - New Entra \\
\hline KCSE & 0.21021 & C & New Entra \\
\hline HP & 0.19833 & A & Survivors \\
\hline AHC & 0.19758 & A & Survivors \\
\hline BRG & 0.19711 & C & New Entra \\
\hline WOL & 0.19365 & A & survivors \\
\hline GOU & 0.19144 & C & New Entra \\
\hline $\begin{array}{l}\text { WISE } \\
\text { ENRGE }\end{array}$ & 0.19025 & $A$ & Survivors \\
\hline POY & 0.18889 & A & - Survivors \\
\hline NLON & 0.18541 & 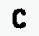 & New Entre \\
\hline SGO & 0.18260 & & New Entra \\
\hline & & & \\
\hline
\end{tabular}


Appendix B

Firm-Specific Efficiency Measures

For Group A (Survivors) and Group C (New Entrants) - 1989-1992

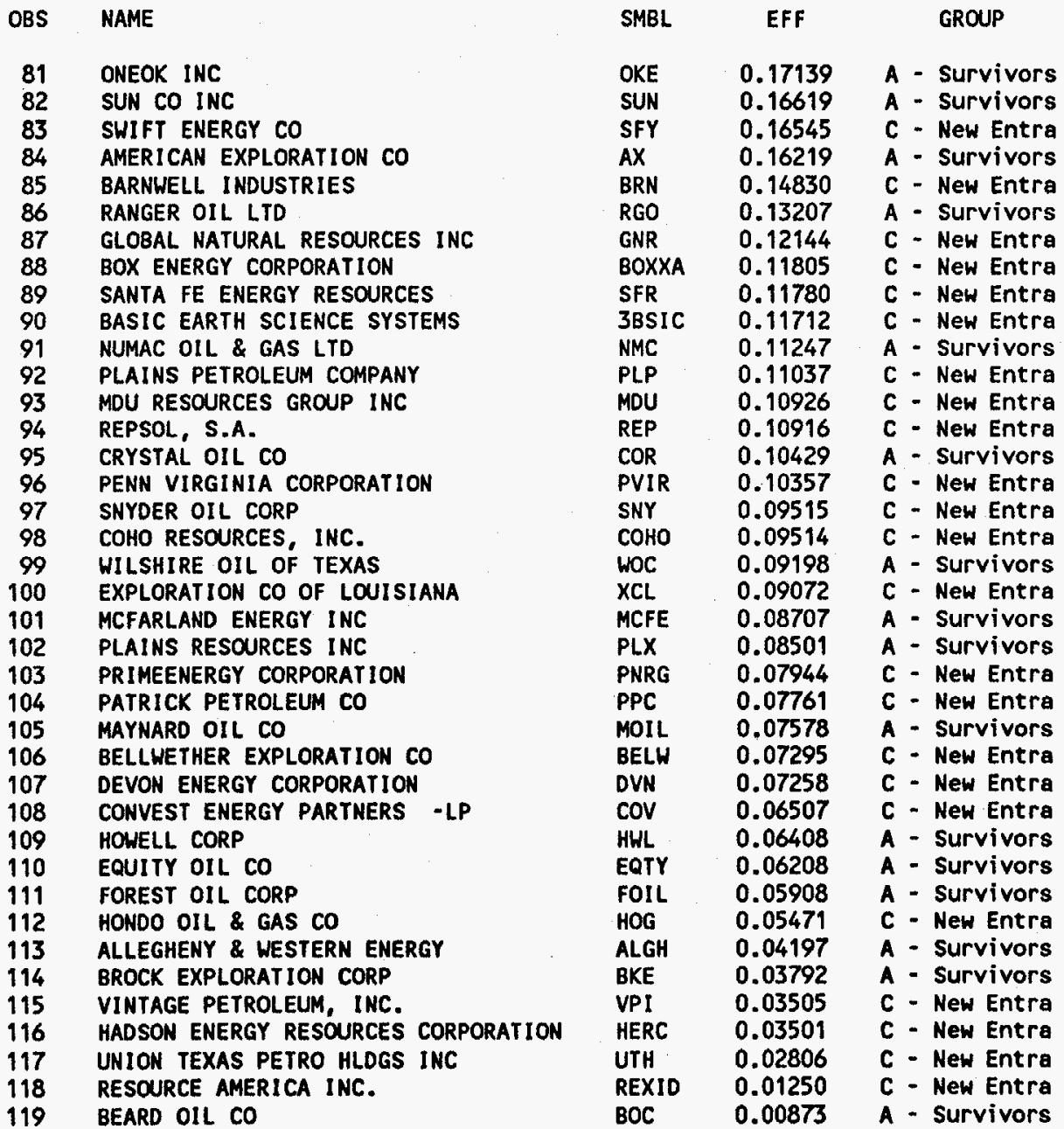


APPENDIX C

MEAN EFFICIENCY MEASURES

41 
Appendix C

For Group A (Survivors) and Group B (Non-survivors) - 1982-1985

Analysis Variable : EFF

GROUP=B- Non-survivors-

\begin{tabular}{ccccc}
$N$ & Mean & Std Dev & Minimum & Maximum \\
\hline 146 & 0.0680822 & 0.0851479 & 0.0023458 & 0.6000051
\end{tabular}

GROUP=A - Survivors

\begin{tabular}{ccccc}
$N$ & Mean & Std Dev & Minimum & Maximum \\
\hline 66 & 0.1473796 & 0.1602076 & 0.0147193 & 1.0000000
\end{tabular}


Appendix C

Mean Efficiency Measures

$09: 38$ Sunday September 25,1994

For Group A (Survivors) and Group C (New Entrants) - 1989-1992

Analysis Variable : EFF

GROUP=A - Survivor

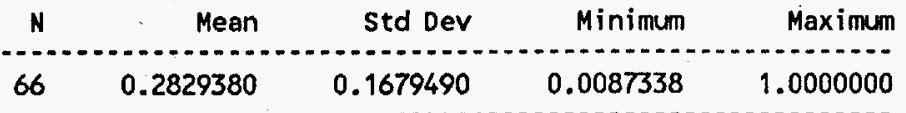

\begin{tabular}{|ccccc} 
& & & & \\
$N$ & Mean & Std Dev & Minimum & Maximum \\
\hline 53 & 0.2537273 & 0.2100844 & 0.0124997 & 0.9836801
\end{tabular}


Appendix C

Test of Difference in Mean Efficiency

For Group A (Survivors) and Group B (Non-survivors) - 1982-1985

Intercept Defined as Group B Mean Efficiency

Model: MODEL 1

Dependent Variable: EFF

\section{EFFICIENCY MEASURE}

\section{Analysis of Variance}

$\begin{array}{lrrrrr}\text { Source } & \text { DF } & \begin{array}{r}\text { Sum of } \\ \text { Squares }\end{array} & \begin{array}{r}\text { Mean } \\ \text { Square }\end{array} & \text { F Value } & \text { Prob>F } \\ \text { Model } & 1 & 0.85743 & 0.85743 & 66.629 & 0.0001 \\ \text { Error } & 210 & 8.15878 & 0.01287 & & \\ \text { C Total } & 211 & 9.01622 & & & \\ & & & & \\ \quad \text { Root MSE } & 0.11344 & \text { R-square } & 0.0951 & \\ \text { Dep Mean } & 0.09277 & \text { Adj R-sq } & 0.0937 & \\ \text { C.V. } & 122.28258 & & & & \end{array}$

Parameter Estimates

\begin{tabular}{|c|c|c|c|c|c|c|c|}
\hline ble & DF & $\begin{array}{r}\text { Parameter } \\
\text { Estimate }\end{array}$ & $\begin{array}{r}\text { Standard } \\
\text { Error }\end{array}$ & $\begin{array}{c}T \text { for } H 0: \\
\text { Parameter }=0\end{array}$ & Prob > $|T|$ & $\begin{array}{r}\text { Variable } \\
\text { Label }\end{array}$ & \\
\hline $\begin{array}{l}\text { NTERCEP } \\
\text { ROUPA }\end{array}$ & $\begin{array}{l}1 \\
1\end{array}$ & $\begin{array}{l}0.068082 \\
0.079297\end{array}$ & $\begin{array}{l}0.00542040 \\
0.00971465\end{array}$ & $\begin{array}{r}12.560 \\
8.163\end{array}$ & $\begin{array}{l}0.0001 \\
0.0001\end{array}$ & $\begin{array}{l}\text { Intercept } \\
\text { DUMMY VARIABLE }\end{array}$ & IND I CATING \\
\hline
\end{tabular}




$$
\text { Appendix } \mathrm{C}
$$

Test of Difference in Mean Efficiency

For Group A (Survivors) and Group C (New Entrants) - 1989-1992

Intercept Defined as Group C Mean Efficiency

Model : MODEL 1

Dependent Variable: EFF

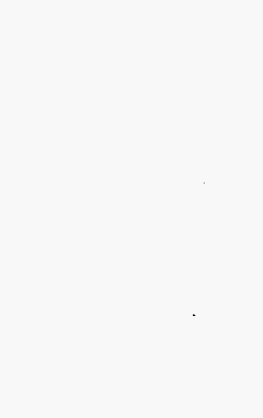

\section{EFFICIENCY MEASURE}

Analysis of Variance

\begin{tabular}{|c|c|c|c|c|c|}
\hline Source & $D F$ & $\begin{array}{l}\text { Sum of } \\
\text { squares }\end{array}$ & $\begin{array}{r}\text { Mean } \\
\text { Square }\end{array}$ & F Value & Prob $>F$ \\
\hline $\begin{array}{l}\text { Model } \\
\text { Error } \\
\text { C Total }\end{array}$ & $\begin{array}{r}1 \\
117 \\
118\end{array}$ & $\begin{array}{r}0.07524 \\
12.38547 \\
12.46071\end{array}$ & $\begin{array}{l}0.07524 \\
0.03489\end{array}$ & 2.157 & 0.1428 \\
\hline $\begin{array}{l}\text { Root MSE } \\
\text { Dep Mean } \\
\text { C.V. }\end{array}$ & & $\begin{array}{l}8679 \\
6993 \\
9805\end{array}$ & $\begin{array}{l}R \text {-square } \\
\text { Adj } R \text {-sq }\end{array}$ & $\begin{array}{l}0.0060 \\
0.0032\end{array}$ & \\
\hline
\end{tabular}

Parameter Estimates

\begin{tabular}{|c|c|c|c|c|c|c|c|}
\hline Variable & DF & $\begin{array}{l}\text { Parameter } \\
\text { Estimate }\end{array}$ & $\begin{array}{r}\text { Standard } \\
\text { Error }\end{array}$ & $\begin{array}{c}T \text { for HO: } \\
\text { Parameter }=0\end{array}$ & Prob > $|T|$ & $\begin{array}{r}\text { Variable } \\
\text { Label }\end{array}$ & \\
\hline $\begin{array}{l}\text { INTERCEP } \\
\text { GROUPA }\end{array}$ & $\begin{array}{l}1 \\
1\end{array}$ & $\begin{array}{l}0.253727 \\
0.029211\end{array}$ & $\begin{array}{l}0.01481302 \\
0.01989047\end{array}$ & $\begin{array}{r}17.129 \\
1.469\end{array}$ & $\begin{array}{l}0.0001 \\
0.1428\end{array}$ & $\begin{array}{l}\text { Intercept } \\
\text { DUMMY VARIABLE }\end{array}$ & INDICATING GROUP A \\
\hline
\end{tabular}




\author{
APPENDIX D \\ SPEARMAN AND PEARSON PRODUCT MOMENT CORRELATION COEFFICIENTS \\ BETWEEN INVERSE FINDING COSTS \\ AND FIRM-SPECIFIC EFFICIENCY MEASURE \\ ANALYSIS POOLING ACROSS YEARS
}


Appendix D

17:51 Sunday, September 25, 199459

Spearman Correlation Coefficients Between EFF and Finding Costs

For Group A (Survivors) and Group B (Non-survivors) - 1982-1985

Analysis Across Years

Correlation Analysis
9 'VAR' Variables:
EFF
IFC1
IFC3
IFC1R
IFC3R
IFC10
IFC3D
IFC1RD
IFC3RD

Variable

EFF

IFC]

I FC3

IFCIR

IFC $3 R$

IFC1D

IFC3D

I FC1RD

IFC3RD simple Statistics

0.09277

1.07128

0.50869

0.38417

0.35085

0.15195

0.13176

0.07426

0.07426

Std Dev

0.11916

5.91587

1.14172

6.83670

1.11260

0.31897

0.12291

.51674

0.19887
Median Minimum

0.05627

0.24467

0.00235

0.00144

0.23926

0.01370

$0.22962-130.15385$

$0.20902 \quad-9.67640$

$0.09576 \quad 0.00122$

$0.10180 \quad 0.00572$

$0.10001-6.86338$

$-2.34284$
$0.10001-6.86338$
Maximum Label

1.00000 EFFICIENCY MEASURE

28.50000 INV. 1-YR FND CSTS W/EXP EXCL. REV

20.70370 INV. 3-YR FND CSTS W/EXP EXCL. REV.

66.08333 INV. 1-YR FND CSTS W/EXP INCL. REV.

13.60435 INV. 3-YR FND CSTS H/EXP INCL. REV.

6.94595 INV. 1-YR FND CSTS H/EXP, DEV EXCL. REV.

1.16823 INV. 3-YR FND CSTS W/EXP, DEV EXCL. REV.

3.57207 INV. 1-YR FND CSTS W/EXP,DEV INCL. REV.

1.51139 INV. 3-YR FND CSTS W/EXP,DEV INCL. REV. 
Appendix $D$

$17: 51$ sunday, September 25, 199460 Spearman Correlation Coefficients Between EFF and Finding Costs

For Group A (Survivors) and Group B (Non-survivors) - 1982-1985

Analysis Across Years

\section{Correlation Analysis}

Spearman Correlation Coefficients / Prob $>|R|$ under Ho: Rho=0 / N $=636$

EFF

EFFICIENCY MEASURE

IFC1

IFC3

INV. 3-YR FND CSTS W/EXP EXCL. REV.

IFCIR

IFCIR
INV. 1 -YR FND CSTS W/EXP INCL. REV.

IFC3R

INV. 3-YR FND CSTS W/EXP INCL. REV.

IFC10

INV. 1-YR FND CSTS W/EXP,DEV EXCL. REV.

IFC30

INV. 3-YR FND CSTS W/EXP, DEV EXCL. REV.

IFCIRD

INY. 1-YR FND CSTS W/EXP,DEV INCL. REV.

IFC3RD

INV. 3-YR FND CSTS W/EXP,DEV INCL. REV.

\begin{tabular}{|c|c|c|c|c|c|c|c|c|}
\hline EFF & IFC1 & I FC3 & IFCIR & IFC3R & IFC1D & IFC3D & IFC1RD & IFC3RD \\
\hline $\begin{array}{l}0000 \\
0\end{array}$ & $\begin{array}{r}0.16595 \\
0.0001\end{array}$ & $\begin{array}{r}0.09269 \\
0.0194\end{array}$ & $\begin{array}{r}0.24498 \\
0.0001\end{array}$ & $\begin{array}{r}0.30164 \\
0.0001\end{array}$ & $\begin{array}{r}0.20723 \\
0.0001\end{array}$ & $\begin{array}{r}0.09722 \\
0.0142\end{array}$ & $\begin{array}{r}0.25776 \\
0.0001\end{array}$ & $\begin{array}{r}0.32617 \\
0.0001\end{array}$ \\
\hline $\begin{array}{l}6595 \\
0001\end{array}$ & $\begin{array}{l}1.00000 \\
0.0\end{array}$ & $\begin{array}{r}0.71498 \\
0.0001\end{array}$ & $\begin{array}{r}0.57692 \\
0.0001\end{array}$ & $\begin{array}{r}0.46893 \\
0.0001\end{array}$ & $\begin{array}{r}0.85441 \\
0.0001\end{array}$ & $\begin{array}{r}0.61362 \\
0.0001\end{array}$ & $\begin{array}{r}0.42871 \\
0.0001\end{array}$ & $\begin{array}{r}0.34486 \\
0.0001\end{array}$ \\
\hline $\begin{array}{l}19269 \\
0194\end{array}$ & $\begin{array}{r}0.71498 \\
0.0001\end{array}$ & $\begin{array}{c}1.00000 \\
0.0\end{array}$ & $\begin{array}{r}0.31022 \\
0.0001\end{array}$ & $\begin{array}{r}0.54997 \\
0.0001\end{array}$ & $\begin{array}{r}0.57134 \\
0.0001\end{array}$ & $\begin{array}{r}0.87097 \\
0.0001\end{array}$ & $\begin{array}{r}0.15709 \\
0.0001\end{array}$ & $\begin{array}{r}0.37807 \\
0.0001\end{array}$ \\
\hline $\begin{array}{l}14498 \\
0001\end{array}$ & $\begin{array}{r}0.57692 \\
0.0001\end{array}$ & $\begin{array}{r}0.31022 \\
0.0001\end{array}$ & $\begin{array}{l}1.00000 \\
0.0\end{array}$ & $\begin{array}{r}0.56774 \\
0.0001\end{array}$ & $\begin{array}{r}0.53547 \\
0.0001\end{array}$ & $\begin{array}{r}0.23339 \\
0.0001\end{array}$ & $\begin{array}{r}0.90920 \\
0.0001\end{array}$ & $\begin{array}{r}0.52866 \\
0.0001\end{array}$ \\
\hline $\begin{array}{l}0164 \\
0001\end{array}$ & $\begin{array}{r}0.46893 \\
0.0001\end{array}$ & $\begin{array}{r}0.54997 \\
0.0001\end{array}$ & $\begin{array}{r}0.56774 \\
0.0001\end{array}$ & $\begin{array}{l}1.00000 \\
0.0\end{array}$ & $\begin{array}{r}0.37698 \\
0.0001\end{array}$ & $\begin{array}{r}0.46040 \\
0.0001\end{array}$ & $\begin{array}{r}0.48535 \\
0.0001\end{array}$ & $\begin{array}{r}0.90632 \\
0.0001\end{array}$ \\
\hline $\begin{array}{l}0723 \\
0001\end{array}$ & $\begin{array}{r}0.85441 \\
0.0001\end{array}$ & $\begin{array}{r}0.57134 \\
0.0001\end{array}$ & $\begin{array}{r}0.53547 \\
0.0001\end{array}$ & $\begin{array}{r}0.37698 \\
0.0001\end{array}$ & $\begin{array}{l}1.00000 \\
0.0\end{array}$ & $\begin{array}{r}0.63799 \\
0.0001\end{array}$ & $\begin{array}{r}0.53120 \\
0.0001\end{array}$ & $\begin{array}{r}0.35991 \\
0.0001\end{array}$ \\
\hline $\begin{array}{l}9722 \\
0142\end{array}$ & $\begin{array}{r}0.61362 \\
0.0001\end{array}$ & $\begin{array}{r}0.87097 \\
0.0001\end{array}$ & $\begin{array}{r}0.23339 \\
0.0001\end{array}$ & $\begin{array}{r}0.46040 \\
0.0001\end{array}$ & $\begin{array}{r}0.63799 \\
0.0001\end{array}$ & $\begin{array}{l}1.00000 \\
0.0\end{array}$ & $\begin{array}{r}0.18104 \\
0.0001\end{array}$ & $\begin{array}{r}0.44050 \\
0.0001\end{array}$ \\
\hline $\begin{array}{l}5776 \\
0001\end{array}$ & $\begin{array}{r}0.42871 \\
0.0001\end{array}$ & $\begin{array}{r}0.15709 \\
0.0001\end{array}$ & $\begin{array}{r}0.90920 \\
0.0001\end{array}$ & $\begin{array}{r}0.48535 \\
0.0001\end{array}$ & $\begin{array}{r}0.53120 \\
0.0001\end{array}$ & $\begin{array}{r}0.18104 \\
0.0001\end{array}$ & $\begin{array}{l}1.00000 \\
0.0\end{array}$ & $\begin{array}{r}0.53485 \\
0.0001\end{array}$ \\
\hline & $\begin{array}{r}0.34486 \\
0.0001\end{array}$ & $\begin{array}{r}0.37807 \\
0.0001\end{array}$ & $\begin{array}{r}0.52866 \\
0.0001\end{array}$ & $\begin{array}{r}0.90632 \\
0.0001\end{array}$ & $\begin{array}{r}0.35991 \\
0.0001\end{array}$ & $\begin{array}{r}0.44050 \\
0.0001\end{array}$ & $\begin{array}{r}0.53485 \\
0.0001\end{array}$ & $\begin{array}{c}1.00000 \\
0.0\end{array}$ \\
\hline
\end{tabular}


Appendix D

17:51 Sunday, September 25, 199461

Spearman Correlation Coefficients Between EFF and Finding Costs

For Group A (Survivors) and Group C (New Entrants) - 1989-1992

Analysis Across Years

Correlation Analysis

9 'VAR' Variables: EFF IFC1 IFC3 IFC1R IFC3R IFC1D IFC3D IFCIRD IFC3RD

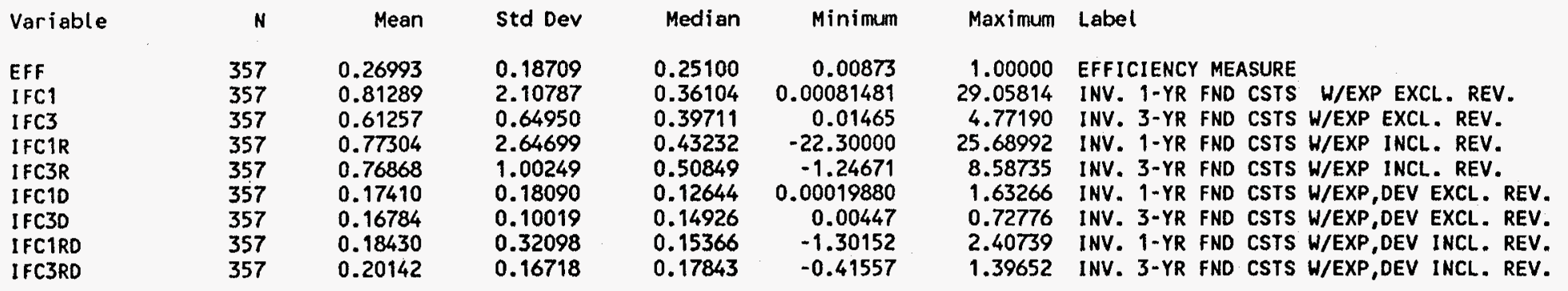


Appendix D

17:51 Sunday, September 25, 199462

Spearman Correlation Coefficients Between EFF and Finding Costs

For Group A (Survivors) and Group C (New Entrants) - 1989-1992

Analysis Across Years

Correlation Analysis

Spearman Correlation Coefficients / Prob > $|R|$ under Ho: Rho=0 / N $=357$

EFF

\section{EFFICIENCY MEASURE}

IFCI

INV. 1-YR FNO CSTS W/EXP EXCL. REV.

IFC3

INV. 3-YR FNO CSTS W/EXP EXCL. REV.

IFCIR

INV. 1-YR FND CSTS W/EXP INCL. REV.

IFC3R

INV. 3-YR FND CSTS W/EXP INCL. REV.

IFC10

INV. 1-YR FND CSTS W/EXP, DEV EXCL. REV.

IFC3D

INV. 3-YR FND CSTS W/EXP,DEV EXCL. REV.

IFCIRD

INV. 1-YR FND CSTS W/EXP,DEV INCL. REV.

IFC3RD

INV. 3-YR FND CSTS W/EXP,DEV INCL. REV.
IFCI IFCS

$\begin{array}{lll}1.00000 & 0.36216 & 0.30754\end{array}$

$\begin{array}{crr}1.00000 & 0.36216 & 0.30754 \\ 0.0 & 0.0001 & 0.0001\end{array}$

$0.36216 \quad 1.00000$

0.0001

0.30754

0.0001

0.0

0.77292

0.77292
0.0001

IFCIR

IFC3R

IFC10

0.34803

0.32612

0.41947

0.70113

0.0001

0.0001

0.41074

0.41074

0.37091

0.0001

0.34803

0.772921 .00000

0.0001

0.62993
0.0001

0.81118
0.0001

0.65477

0.53428
0.0001

0.0001

0.34803
0.0001

0.70113

0.0

.51550

0.72722

0.53582
0.0001

0.0001

0.32612

0.0001

0.51550
0.0001

1,00000

0.0001

0.77825

0.30394

0.53028

0.0001

0.62993

0.72722

0.0

0.68802

0.50315

0.33605

0.33605
0.0001

0.84132

0.58735

0.0001

0.0001

0.68802

0.0001

1.00000

0.0

0.36571

0.0001

0.44269

0.0001

0.0001

0.41947
0.0001

0.81118
0.0001

0.53582
0.0001

0.50315
0.0001

0.36571
0.0001

1.00000

0.0001

0.49603

0.0001

0.81132

0.0001

0.41074

0.65477

0.77825

0.0001

0.0001

0.49730
0.0001

0.0001

0.0001

0.0001

0.33605

0.44269

0.72746

1.00000

0.36480

0.37091

0.53428

0.30394

0.84132

0.0001

0.0001

0.0

0.0001

0.57239

0.42158

0.42158
0.0001

0.84132
0.0001

0.49603
0.0001

0.61229

1.00000

0.63313

0.51394

0.53028

0.58735

0.81132

0.49730

0.0

0.0001

0.0001

0.0001

0.0001

0.0001

0.0001

0.63313
0.0001

0.0 
Appendix $D$

17:51 Sunday, September 25, 199463

Pearson Correlation Coefficients Between EFF and Finding Costs

For Group A (Survivors) and Group B (Non-survivors) - 1982-1985

Analys is Across Years

Correlation Analysis

9 'VAR' Variables: EFF IFC1 IFC3 IFC1R IFC3R IFC1D IFC3D IFC1RD IFC3RD

Simple Statistics

Variable

EFF

IFC1

IFC3

IFC3R

$I F C 3 R$

IFCID

1 FC30

IFCIRD

IFC3RD
Mean

0.09277

1.07128

0.50869

0.38417

0.35085

0.15195

0.13176

0.13176

0.07426

0.09475
Std Dev

0.11916

5.91587

1.14172

6.83670

1.11260

0.31897

0.12291

0.51674

0.19887 sum

59.00117

681.33431

323.52780

244.32950

223.14360

96.63917

83.79798

47.23060

60.26042

\section{Minimum}

0.00235

0.00144 0.01370

$-130.15385$

$-9.67640$

00122

0.00572

$-6.86338$

$-2.34284$

\section{Maximum Label}

1.00000 EFFICIENCY MEASURE

128.50000 INV. 1-YR FND CSTS W/EXP EXCL. REV.

20.70370 INV. 3-YR FND CSTS W/EXP EXCL. REV.

66.08333 INV. 1-YR FND CSTS W/EXP INCL. REV.

13.60435 INV. 3-YR FND CSTS W/EXP INCL. REV.

6.94595 INV. 1-YR FND CSTS W/EXP, DEV EXCL. REV.

1.16823 INV. 3-YR FND CSTS W/EXP, DEV EXCL. REV.

3.57207 INV 1-YR FND CSTS W/EXP DEV INCL. REV.

1.51139 INV. 3-YR FND CSTS W/EXP,DEV INCL. REV. 
Appendix D

17:51 Sunday, September 25, 199464 Pearson Correlation Coefficients Between EFF and Finding Costs For Group A (Survivors) and Group B (Non-survivors) - 1982-1985

Analysis Across Years

Correlation Analysis

Pearson Correlation Coefficients / Prob > $|R|$ under Ho: Rho $=0 / N=636$

EFF

EFFICIENCY MEASURE

IFC1

INV. 1-YR FND CSTS W/EXP EXCL. REV.

IFC3

INV. 3-YR FND CSTS W/EXP EXCL. REV.

IFCIR

INV. 1-YR FND CSTS W/EXP INCL. REV.

IFC3R

INV. 3-YR FND CSTS H/EXP INCL. REV.

IFC10

INV. 1-YR FND CSTS W/EXP,DEV EXCL. REV.

IFC30

INV. 3-YR FND CSTS H/EXP,DEV EXCL. REV.

IFCIRD

INV. 1-YR FND CSTS W/EXP,DEV INCL. REV.

IFC3RO

INV. 3-YR FND CSTS W/EXP,DEV INCL. REV.

$\begin{array}{rrrrrrrrrr}\text { EFF } & \text { IFC1 } & \text { IFC3 } & \text { IFC1R } & \text { IFC3R } & \text { IFC1D } & \text { IFC3D } & \text { IFC1RD } & \text { IFC3RD } \\ 1.00000 & -0.03329 & -0.04161 & -0.01702 & 0.02015 & -0.02860 & -0.04131 & 0.03261 & 0.08463 \\ 0.0 & 0.4019 & 0.2947 & 0.6684 & 0.6119 & 0.4716 & 0.2982 & 0.4116 & 0.0328 \\ -0.03329 & 1.00000 & 0.77591 & 0.32621 & 0.44328 & 0.82695 & 0.43315 & 0.26523 & 0.14859 \\ 0.4019 & 0.0 & 0.0001 & 0.0001 & 0.0001 & 0.0001 & 0.0001 & 0.0001 & 0.0002 \\ -0.04161 & 0.77591 & 1.00000 & 0.29157 & 0.63392 & 0.74321 & 0.70924 & 0.13792 & 0.24259 \\ 0.2947 & 0.0001 & 0.0 & 0.0001 & 0.0001 & 0.0001 & 0.0001 & 0.0005 & 0.0001 \\ -0.01702 & 0.32621 & 0.29157 & 1.00000 & 0.36002 & 0.38153 & 0.16962 & 0.41579 & 0.26525 \\ 0.6684 & 0.0001 & 0.0001 & 0.0 & 0.0001 & 0.0001 & 0.0001 & 0.0001 & 0.0001 \\ 0.02015 & 0.44328 & 0.63392 & 0.36002 & 1.00000 & 0.35887 & 0.41721 & 0.53777 & 0.77557 \\ 0.6119 & 0.0001 & 0.0001 & 0.0001 & 0.0 & 0.0001 & 0.0001 & 0.0001 & 0.0001 \\ -0.02860 & 0.82695 & 0.74321 & 0.38153 & 0.35887 & 1.00000 & 0.55092 & 0.29490 & 0.14184 \\ 0.4716 & 0.0001 & 0.0001 & 0.0001 & 0.0001 & 0.0 & 0.0001 & 0.0001 & 0.0003 \\ -0.04131 & 0.43315 & 0.70924 & 0.16962 & 0.41721 & 0.55092 & 1.00000 & -0.07443 & 0.25672 \\ 0.2982 & 0.0001 & 0.0001 & 0.0001 & 0.0001 & 0.0001 & 0.0 & 0.0607 & 0.0001 \\ 0.03261 & 0.26523 & 0.13792 & 0.41579 & 0.53777 & 0.29490 & -0.07443 & 1.00000 & 0.61109 \\ 0.4116 & 0.0001 & 0.0005 & 0.0001 & 0.0001 & 0.0001 & 0.0607 & 0.0 & 0.0001 \\ 0.08463 & 0.14859 & 0.24259 & 0.26525 & 0.77557 & 0.14184 & 0.25672 & 0.61109 & 1.00000 \\ 0.0328 & 0.0002 & 0.0001 & 0.0001 & 0.0001 & 0.0003 & 0.0001 & 0.0001 & 0.0\end{array}$


Appendix D

17:51 Sunday, September 25, 199465

Pearson Correlation Coefficients Between EFF and Finding costs

For Group A (Survivors) and Group C (New Entrants) - 1989-1992

Analys is Across Years

Correlation Analysis

9 'VAR' Variables: EFF

IFC1

IFC3

IFCIR IFC3R

IFC1D

IFC3D

IFC1RD

IFC3RD

simple Statistics

$\begin{array}{lrrrrr}\text { Variable } & \text { N } & \text { Mean } & \text { Std Dev } & \text { Sum } & \text { Minimum } \\ \text { EFF } & & & & & \\ \text { IFC1 } & 357 & 0.26993 & 0.18709 & 96.36436 & 0.00873 \\ \text { IFC3 } & 357 & 0.81289 & 2.10787 & 290.20329 & 0.00081481 \\ \text { IFC1R } & 357 & 0.61257 & 0.64950 & 218.68697 & 0.01465 \\ \text { IFC3R } & 357 & 0.77304 & 2.64699 & 275.97375 & -22.30000 \\ \text { IFC10 } & 357 & 0.76868 & 1.00249 & 274.41913 & -1.24671 \\ \text { IFC3D } & 357 & 0.17410 & 0.18090 & 62.15322 & 0.00019880 \\ \text { IFC1RD } & 357 & 0.16784 & 0.10019 & 59.91721 & 0.00447 \\ \text { IFC3RD } & 357 & 0.18430 & 0.32098 & 65.79584 & -1.30152 \\ & 357 & 0.20142 & 0.16718 & 71.90617 & -0.41557\end{array}$

\section{Maximum Label}

1.00000 EFFICIENCY MEASURE

29.05814 INV. 1-YR FND CSTS W/EXP EXCL. REV.

4.77190 INV. 3-YR FND CSTS W/EXP EXCL. REV.

25.68992 INV. 1-YR FND CSTS W/EXP INCL. REV

8.58735 INV. 3-YR FND CSTS H/EXP INCL. REV.

1.63266 INV. 1-YR FND CSTS W/EXP,DEV EXCL. REV.

0.72776 INV. 3-YR FND CSTS W/EXP,DEV EXCL. REV

2.40739 INV. 1-YR FND CSTS W/EXP,DEV INCL. REV

1.39652 INV. 3-YR FND CSTS W/EXP,DEV INCL. REV. 
Appendix D

17:51 Sunday, September 25, 199466 Pearson Correlation Coefficients Between EFF and $F$ inding Costs

For Group A (Survivors) and Group C (New Entrents) - 1989-1992

Analys is Across Years

Correlation Analysis

Pearson Correlation Coefficients / Prob > $|R|$ under Ho: Rho=0 / N $=357$

EFF

EFFICIENCY MEASURE

IFCI

INV. 1-YR FND CSTS W/EXP EXCL. REV.

IFC3

INV. 3-YR FND CSTS W/EXP EXCL. REV.

IFC1R

INV. I-YR FND CSTS W/EXP INCL. REV.

IFC3R

INV. 3-YR FND CSTS W/EXP INCL. REV.

IFCID

INV. 1-YR FND CSTS W/EXP,DEV EXCL. REV.

IFC3D

INV. 3-YR FND CSTS H/EXP,DEV EXCL. REV.

IFCIRD

INV.

IFC3RD

INV. 3-YR FND CSTS W/EXP,DEV INCL. REV.

\begin{tabular}{|c|c|c|c|c|c|c|c|c|}
\hline EFF & IFCI & IFC3 & IFC1R & IFC3R & IFC1D & IFC3D & IFCIRD & I FC3RD \\
\hline $\begin{array}{l}00000 \\
.0\end{array}$ & $\begin{array}{r}0.09535 \\
0.0720\end{array}$ & $\begin{array}{r}0.14355 \\
0.0066\end{array}$ & $\begin{array}{r}0.13697 \\
0.0096\end{array}$ & $\begin{array}{r}0.09 \\
0.0\end{array}$ & $\begin{array}{r}0.263 \\
0.00\end{array}$ & $\begin{array}{r}0.34737 \\
0.0001\end{array}$ & $\begin{array}{r}0.24 \\
0.0\end{array}$ & $\begin{array}{r}0.30788 \\
0.0001\end{array}$ \\
\hline $\begin{array}{l}59535 \\
.0720\end{array}$ & $\begin{array}{l}1.00000 \\
0.0\end{array}$ & $\begin{array}{r}0.58109 \\
0.0001\end{array}$ & $\begin{array}{r}0.65 \\
0.0\end{array}$ & $\begin{array}{r}0.315 \\
0.00\end{array}$ & $\begin{array}{r}0.52365 \\
0.0001\end{array}$ & $\begin{array}{r}0.35758 \\
0.0001\end{array}$ & $\begin{array}{c}0.2 \\
0 .\end{array}$ & $\begin{array}{r}0.19005 \\
0.0003\end{array}$ \\
\hline $\begin{array}{l}14355 \\
.0066\end{array}$ & $\begin{array}{r}0.58109 \\
0.0001\end{array}$ & $\begin{array}{c}1.00000 \\
0.0\end{array}$ & $\begin{array}{r}0.37753 \\
0.0001\end{array}$ & $\begin{array}{r}0.69683 \\
0.0001\end{array}$ & $\begin{array}{r}0.44944 \\
0.0001\end{array}$ & $\begin{array}{r}0.59066 \\
0.0001\end{array}$ & $\begin{array}{r}0.22253 \\
0.0001\end{array}$ & $\begin{array}{r}0.36343 \\
0.0001\end{array}$ \\
\hline $\begin{array}{l}13697 \\
.0096\end{array}$ & $\begin{array}{r}0.65151 \\
0.0001\end{array}$ & $\begin{array}{r}0.37753 \\
0.0001\end{array}$ & $\begin{array}{c}1.00000 \\
0.0\end{array}$ & $\begin{array}{r}0.44242 \\
0.0001\end{array}$ & $\begin{array}{r}0.42068 \\
0.0001\end{array}$ & $\begin{array}{r}0.24299 \\
0.0001\end{array}$ & $\begin{array}{r}0.62320 \\
0.0001\end{array}$ & $\begin{array}{r}0.39873 \\
0.0001\end{array}$ \\
\hline $\begin{array}{l}96994 \\
.0673\end{array}$ & $\begin{array}{r}0.31510 \\
0.0001\end{array}$ & $\begin{array}{r}0.69683 \\
0.0001\end{array}$ & $\begin{array}{r}0.44242 \\
0.0001\end{array}$ & $\begin{array}{l}1.00000 \\
0.0\end{array}$ & $\begin{array}{r}0.31024 \\
0.0001\end{array}$ & $\begin{array}{r}0.33668 \\
0.0001\end{array}$ & $\begin{array}{r}0.39768 \\
0.0001\end{array}$ & $\begin{array}{r}0.64047 \\
0.0001\end{array}$ \\
\hline $\begin{array}{l}26354 \\
.0001\end{array}$ & $\begin{array}{r}0.52365 \\
0.0001\end{array}$ & $\begin{array}{r}0.44944 \\
0.0001\end{array}$ & $\begin{array}{r}0.42068 \\
0.0001\end{array}$ & $\begin{array}{r}0.31024 \\
0.0001\end{array}$ & $\begin{array}{c}1.00000 \\
0.0\end{array}$ & $\begin{array}{r}0.72409 \\
0.0001\end{array}$ & $\begin{array}{r}0.66674 \\
0.0001\end{array}$ & $\begin{array}{r}0.52064 \\
0.0001\end{array}$ \\
\hline $\begin{array}{l}34737 \\
.0001\end{array}$ & $\begin{array}{r}0.35758 \\
0.0001\end{array}$ & $\begin{array}{r}0.59066 \\
0.0001\end{array}$ & $\begin{array}{r}0.24299 \\
0.0001\end{array}$ & $\begin{array}{r}0.33668 \\
0.0001\end{array}$ & $\begin{array}{r}0.72409 \\
0.0001\end{array}$ & $\begin{array}{l}1.00000 \\
0.0\end{array}$ & $\begin{array}{r}0.37821 \\
0.0001\end{array}$ & $\begin{array}{r}0.53978 \\
0.0001\end{array}$ \\
\hline $\begin{array}{l}24442 \\
.0001\end{array}$ & $\begin{array}{r}0.27514 \\
0.0001\end{array}$ & $\begin{array}{r}0.22253 \\
0.0001\end{array}$ & $\begin{array}{r}0.62320 \\
0.0001\end{array}$ & $\begin{array}{r}0.39768 \\
0.0001\end{array}$ & $\begin{array}{r}0.66674 \\
0.0001\end{array}$ & $\begin{array}{r}0.37821 \\
0.0001\end{array}$ & $\begin{array}{l}1.00000 \\
0.0\end{array}$ & $\begin{array}{r}0.69702 \\
0.0001\end{array}$ \\
\hline & $\begin{array}{r}0.19005 \\
0.0003\end{array}$ & $\begin{array}{r}0.36343 \\
0.0001\end{array}$ & $\begin{array}{r}0.39873 \\
0.0001\end{array}$ & $\begin{array}{r}0.64047 \\
0.0001\end{array}$ & $\begin{array}{r}0.52 \\
0.0\end{array}$ & $\begin{array}{r}0.53978 \\
0.0001\end{array}$ & $\begin{array}{r}0.69702 \\
0.0001\end{array}$ & $\begin{array}{c}1.00000 \\
0.0\end{array}$ \\
\hline
\end{tabular}




\author{
APPENDIX E \\ SPEARMAN AND PEARSON PRODUCT MOMENT CORRELATION COEFFICIENTS \\ BETWEEN INVERSE FINDING COSTS \\ AND FIRM-SPECIFIC EFFICIENCY MEASURE \\ ANALYSIS YEAR-BY-YEAR
}


INTENTIONALLY BLANK 
Appendix E

Spearman Correlation Coefficients Between EFF and Finding Costs

For Group A (Survivors) and Group B (Non-survivors) - 1982-1985

Analysis By Year

YEAR=83

Correlation Analys is

9 'VAR' Variables:

EFF

IFC3

IFC1R

IFC3R

IFC1D

IFC3D

IFC1RD

IFC3RD

\section{Simple Statistics}

$\begin{array}{lrrrrr}\text { Variable } & \text { N } & \text { Mean } & \text { Std Dev } & \text { Median } & \text { Minimum } \\ \text { EFF } & & & & & \\ \text { IFC1 } & 212 & 0.09277 & 0.11935 & 0.05627 & 0.00235 \\ \text { IFC3 } & 212 & 0.56113 & 1.09954 & 0.24206 & 0.01055 \\ \text { IFC1R } & 212 & 0.47951 & 0.82599 & 0.24224 & 0.01370 \\ \text { IFC3R } & 212 & 0.25631 & 1.68745 & 0.23765 & -13.30195 \\ \text { IFC1D } & 212 & 0.38587 & 0.68317 & 0.20476 & -1.00943 \\ \text { IFC3D } & 212 & 0.13048 & 0.12252 & 0.10343 & 0.00311 \\ \text { IFC1RD } & 212 & 0.13112 & 0.12307 & 0.09842 & 0.00572 \\ \text { IFC3RD } & 212 & 0.07390 & 0.36763 & 0.10747 & -4.24214 \\ & 212 & 0.10789 & 0.12953 & 0.08973 & -0.50540\end{array}$

\section{Maximum Label}

1.00000 EFFICIENCY MEASURE

8.21083 INV. 1-YR FND CSTS W/EXP EXCL. REV.

7.83708 INV. 3-YR FND CSTS W/EXP EXCL. REV.

10.27444 INV. 1-YR FND CSTS W/EXP INCL. REV.

4.33663 INV. 3-YR FND CSTS W/EXP INCL. REV.

0.82873 INV. 1-YR FND CSTS W/EXP,DEV EXCL. REV.

1.04001 INV. 3-YR FND CSTS W/EXP,DEV EXCL. REV.

1.04001 INV. 3-YR FND CSTS W/EXP,DEV EXCL. REV.

0.74520 INV. 3-YR FND CSTS W/EXP,DEV INCL. REV. 
Appendix $E$

Spearman Correlation Coefficients Between EFF and Finding Costs

For Group A (Survivors) and Group B (Non-survivors) - 1982-1985 Analysis By Year

Correlation Analys is

Spearman Correlation Coefficients / Prob > $|R|$ under Ho: Rho=0 / N $=212$

EFF

EFFICIENCY MEASURE

IFC1 1 INV. $1-$ YR FND CSTS W/EXP EXCL. REV.

IFC3

INV. 3-YR FND CSTS W/EXP EXCL. REV.

IFCIR

INV. 1-YR FND CSTS W/EXP INCL. REV.

IFC3R

INV. 3-YR FND CSTS W/EXP INCL. REV

IFC10

INV. 1-YR FND CSTS W/EXP,DEV EXCL. REV.

IFC3D

INV. 3-YR FND CSTS W/EXP, DEV EXCL. REV.

IFC1RD

INV. 1-YR FND CSTS W/EXP,DEV INCL. REV.

IFC3RD

INV. 3-YR FND CSTS W/EXP,DEV INCL. REV.

$\begin{array}{rrrrrrrrrr}\text { EFF } & \text { IFC1 } & \text { IFC3 } & \text { IFC1R } & \text { IFC3R } & \text { IFC1D } & \text { IFC3D } & \text { IFC1RD } & \text { IFC3RD } \\ 1.00000 & 0.10255 & 0.01867 & 0.26592 & 0.19164 & 0.14181 & -0.01322 & 0.28135 & 0.20597 \\ 0.0 & 0.1367 & 0.7870 & 0.0001 & 0.0051 & 0.0391 & 0.8483 & 0.0001 & 0.0026 \\ 0.10255 & 1.00000 & 0.73884 & 0.54079 & 0.57692 & 0.83502 & 0.03147 & 0.39051 & 0.44585 \\ 0.1367 & 0.0 & 0.0001 & 0.0001 & 0.0001 & 0.0001 & 0.0001 & 0.0001 & 0.0001 \\ 0.01867 & 0.73884 & 1.00000 & 0.28021 & 0.66086 & 0.57736 & 0.88832 & 0.12095 & 0.50260 \\ 0.7870 & 0.0001 & 0.0 & 0.0001 & 0.0001 & 0.0001 & 0.0001 & 0.0789 & 0.0001 \\ 0.26592 & 0.54079 & 0.28021 & 1.00000 & 0.52763 & 0.52305 & 0.13749 & 0.89668 & 0.44698 \\ 0.0001 & 0.0001 & 0.0001 & 0.0 & 0.0001 & 0.0001 & 0.0456 & 0.0001 & 0.0001 \\ 0.19164 & 0.57692 & 0.66086 & 0.52763 & 1.00000 & 0.45093 & 0.54582 & 0.41739 & 0.90223 \\ 0.0051 & 0.0001 & 0.0001 & 0.0001 & 0.0 & 0.0001 & 0.0001 & 0.0001 & 0.0001 \\ 0.14181 & 0.83502 & 0.57736 & 0.52305 & 0.45093 & 1.00000 & 0.61248 & 0.54711 & 0.44180 \\ 0.0391 & 0.0001 & 0.0001 & 0.0001 & 0.0001 & 0.0 & 0.0001 & 0.0001 & 0.0001 \\ -0.01322 & 0.63147 & 0.88832 & 0.13749 & 0.54582 & 0.61248 & 1.00000 & 0.07089 & 0.53673 \\ 0.8483 & 0.0001 & 0.0001 & 0.0456 & 0.0001 & 0.0001 & 0.0 & 0.3043 & 0.0001 \\ 0.28135 & 0.39051 & 0.12095 & 0.89668 & 0.41739 & 0.54711 & 0.07089 & 1.00000 & 0.43868 \\ 0.0001 & 0.0001 & 0.0789 & 0.0001 & 0.0001 & 0.0001 & 0.3043 & 0.0 & 0.0001 \\ 0.20597 & 0.44585 & 0.50260 & 0.44698 & 0.90223 & 0.44180 & 0.53673 & 0.43868 & 1.00000 \\ 0.0026 & 0.0001 & 0.0001 & 0.0001 & 0.0001 & 0.0001 & 0.0001 & 0.0001 & 0.0\end{array}$


Appendix $E$

17:51 Sunday, September 25, 199437

Spearman Correlation Coefficients Between EFF and Finding Costs

for Group A (Survivors) and Group B (Non-survivors) - 1982-1985

Analys is By Year

YEAR $=84$

Correlation Analysis

Q 'VAR' Variables: EFF

IFC3

IFC1R

IFC3R

IFC10

IFC30

IFC1RD

IFC3RD

Simple Statistics

$\begin{array}{lrrrrr}\text { Variable } & N & \text { Mean } & \text { Std Dev } & \text { Median } & \text { Minimum } \\ \text { EFF } & & & & & \\ \text { IFC1 } & 212 & 0.09277 & 0.11935 & 0.05627 & 0.00235 \\ \text { IFC3 } & 212 & 0.92626 & 3.50066 & 0.22829 & 0.00144 \\ \text { IFC1R } & 212 & 0.43189 & 0.62247 & 0.22884 & 0.02071 \\ \text { IFC3R } & 212 & 0.99947 & 3.96913 & 0.22275 & -4.10151 \\ \text { IFC1D } & 212 & 0.36730 & 0.84016 & 0.20596 & -1.78176 \\ \text { IFC3D } & 212 & 0.14762 & 0.17671 & 0.09699 & 0.00122 \\ \text { IFC1RD } & 212 & 0.12944 & 0.12001 & 0.09951 & 0.01430 \\ \text { IFC3RD } & 212 & 0.12400 & 0.35784 & 0.09818 & -2.87737 \\ & 212 & 0.10479 & 0.16240 & 0.09361 & -0.88176\end{array}$

Maximum Label

1.00000 EFFICIENCY MEASURE

46.31727 INV. 1-YR FND CSTS W/EXP EXCL. REV.

5.48864 INV. 3-YR FND CSTS W/EXP EXCL. REV.

43.58434 INV. 1-YR FND CSTS W/EXP INCL. REV.

8.41572 INV. 3-YR FND CSTS W/EXP INCL. REV

1.39231 INV. $1-Y R$ FND CSTS W/EXP,DEV EXCL. REV.

0.98571 INV. 3-YR FND CSTS W/EXP,DEV EXCL. REV.

2.41624 INV. 1-YR FNO CSTS H/EXP,DEV INCL. REV.

1.51139 INV. 3-YR FND CSTS W/EXP,DEV INCL. REV. 
Appendix $E$

17:51 Sunday, September 25, 199438

Spearman Correlation Coefficients Between EFF and Finding Costs

For Group A (Survivors) and Group B (Non-survivors) - 1982-1985

Analysis By Year

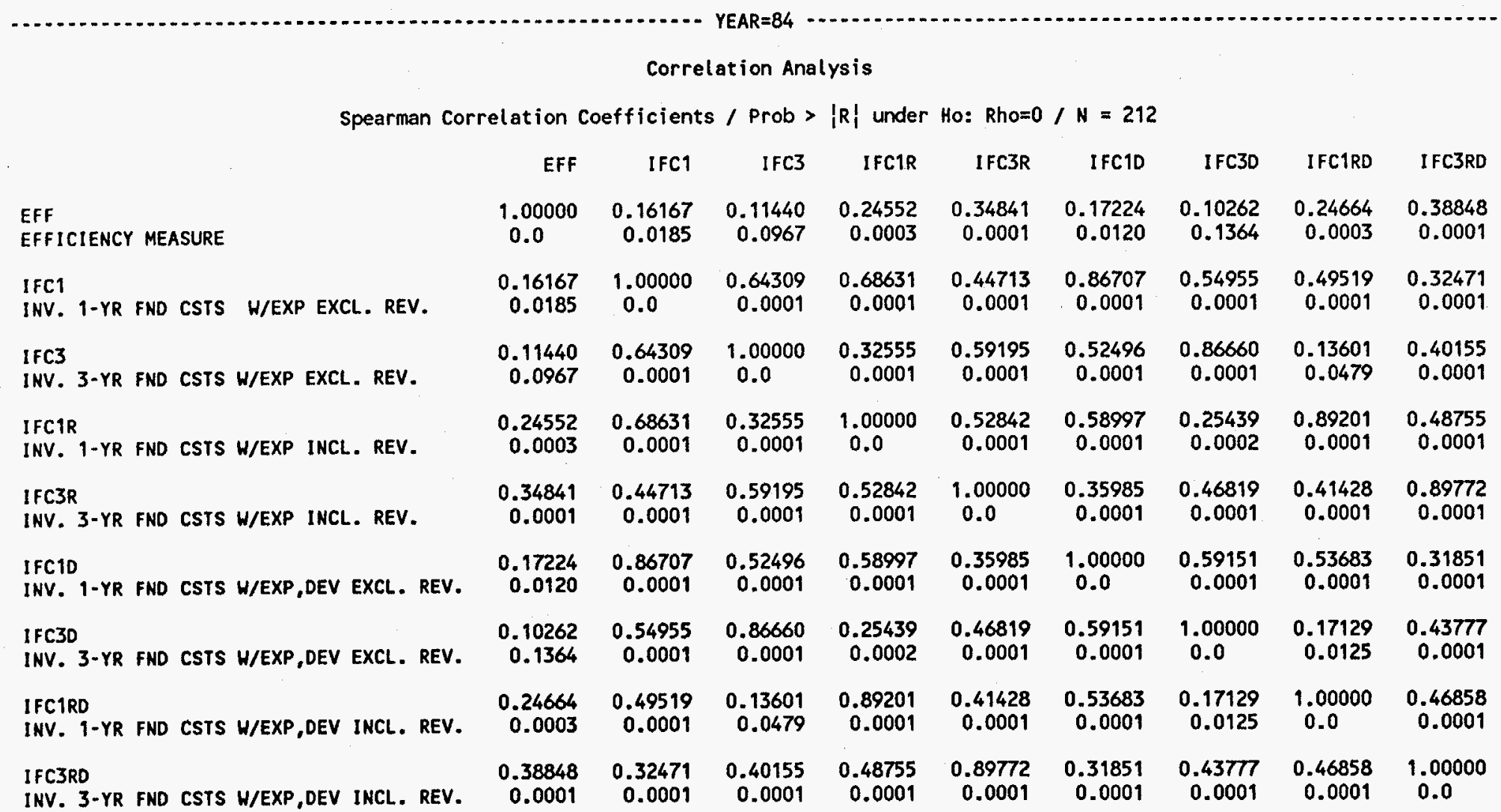


Appendix $E$

Spearman Correlation Coefficients Between EFF and Finding Costs

for Group A (Survivors) and Group B (Non-survivors) - 1982-1985

Analys is By Year

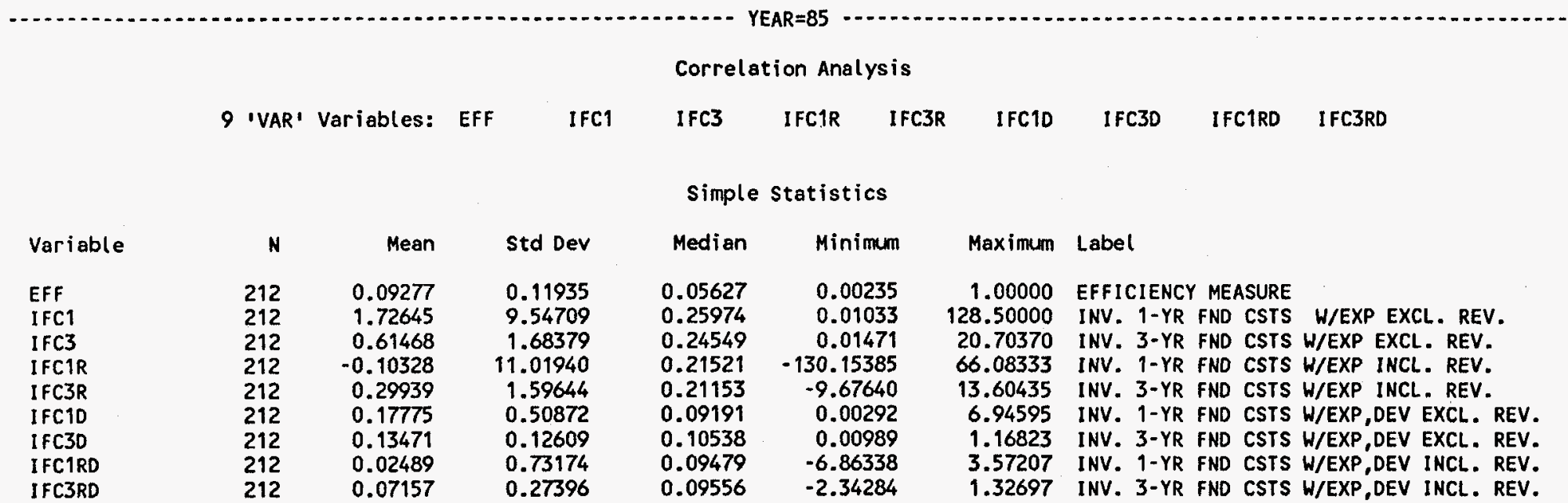


Appendix E

Spearman Correlation Coefficients Between EFF and Finding Costs

For Group A (Survivors) and Group B (Non-survivors) - 1982-1985

Analys is By Year

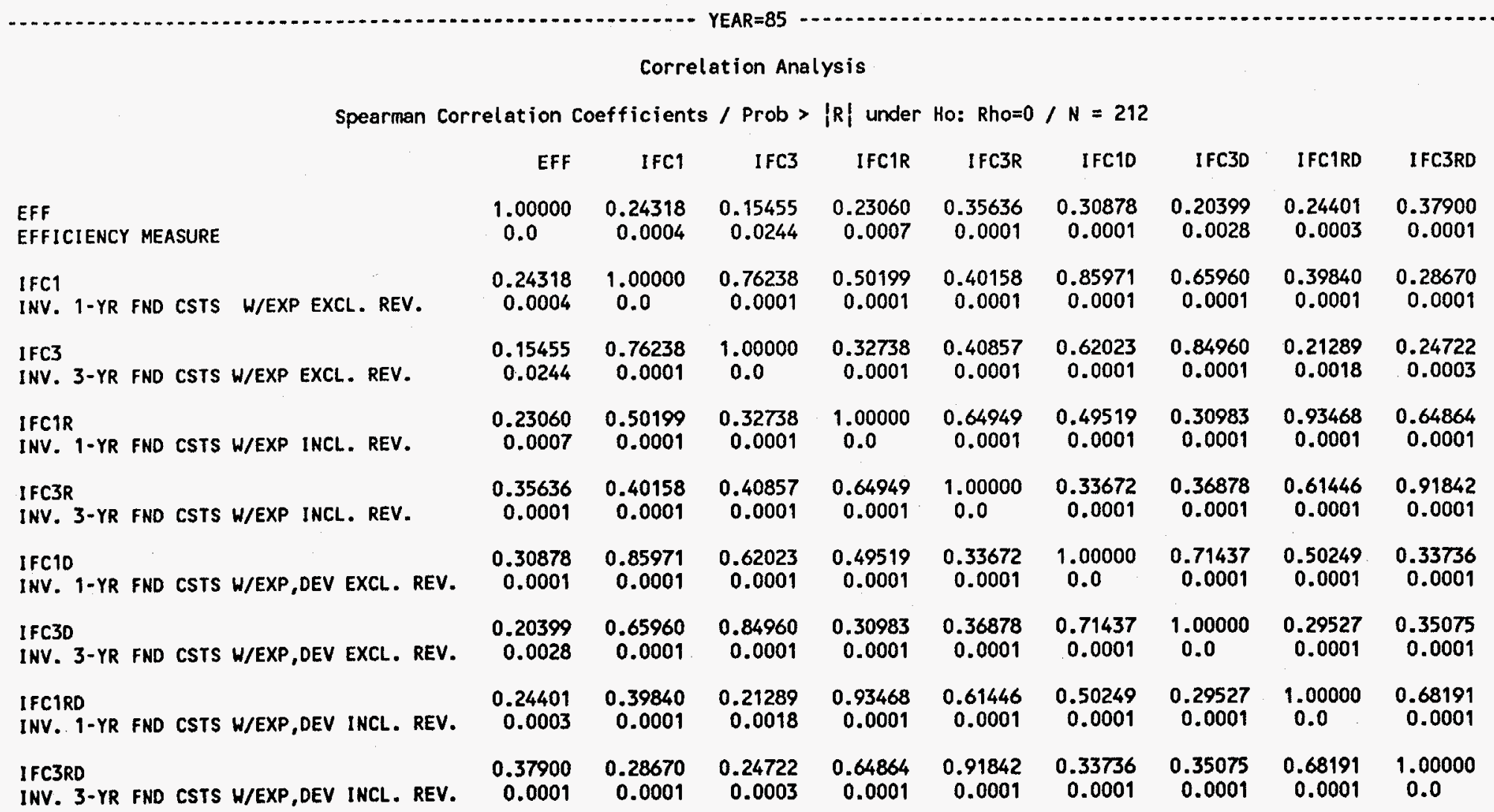


Appendix E

$17: 51$ Sunday, September $25,1994 \quad 41$ Spearman Correlation Coefficients Between EFF and Finding Costs

For Group A (Survivors) and Group C (New Entrants) - 1989-1992

Analys is By Year

YEAR $=90$

Correlation Analysis

9 'VAR' Variables: EFF IFCI IFC3 IFCIR IFC3R IFC1D IFC3D IFC1RD IFC3RD

Simple statistics

$\begin{array}{lrrrrrrl}\text { Variable } & N & \text { Mean } & \text { Std Dev } & \text { Median } & \text { Minimum } & \text { Maximum Label } \\ & & & & & & \\ \text { EFF } & 119 & 0.26993 & 0.18762 & 0.25100 & 0.00873 & 1.00000 \text { EFFICIENCY MEASURE } \\ \text { IFC1 } & 119 & 0.79216 & 1.78106 & 0.36920 & 0.00450 & 18.68301 \text { INV. 1-YR FND CSTS W/EXP EXCL. REV. } \\ \text { IFC3 } & 119 & 0.63950 & 0.68873 & 0.38808 & 0.03101 & 4.77190 \text { INV. 3-YR FND CSTS W/EXP EXCL. REV. } \\ \text { IFC1R } & 119 & 0.98887 & 1.82379 & 0.47746 & -0.85497 & 15.04575 \text { INV. 1-YR FND CSTS W/EXP INCL. REV. } \\ \text { IFC3R } & 119 & 0.91094 & 1.24330 & 0.54796 & -0.29491 & 8.58735 \text { INV. 3-YR FND CSTS W/EXP INCL. REV. } \\ \text { IFC1D } & 119 & 0.18091 & 0.13060 & 0.13780 & 0.00303 & 0.74072 \text { INV. 1-YR FND CSTS W/EXP,DEV EXCL. REV. } \\ \text { IFC3D } & 119 & 0.17419 & 0.10602 & 0.15351 & 0.01624 & 0.59069 \text { INV. 3-YR FND CSTS W/EXP,DEV EXCL. REV. } \\ \text { IFC1RD } & 119 & 0.23033 & 0.25632 & 0.17705 & -0.19099 & 2.11141 \text { INV. 1-YR FND CSTS W/EXP,DEV INCL. REV. } \\ \text { IFC3RD } & 119 & 0.23058 & 0.18048 & 0.19352 & -0.25259 & 1.37928 \text { INV. 3-YR FND CSTS W/EXP,DEV INCL. REV. }\end{array}$


Appendix $E$

Spearman Correlation Coefficients Between EFF and Finding Costs

For Group A (Survivors) and Group C (New Entrants) - 1989-1992

Analysis By Year

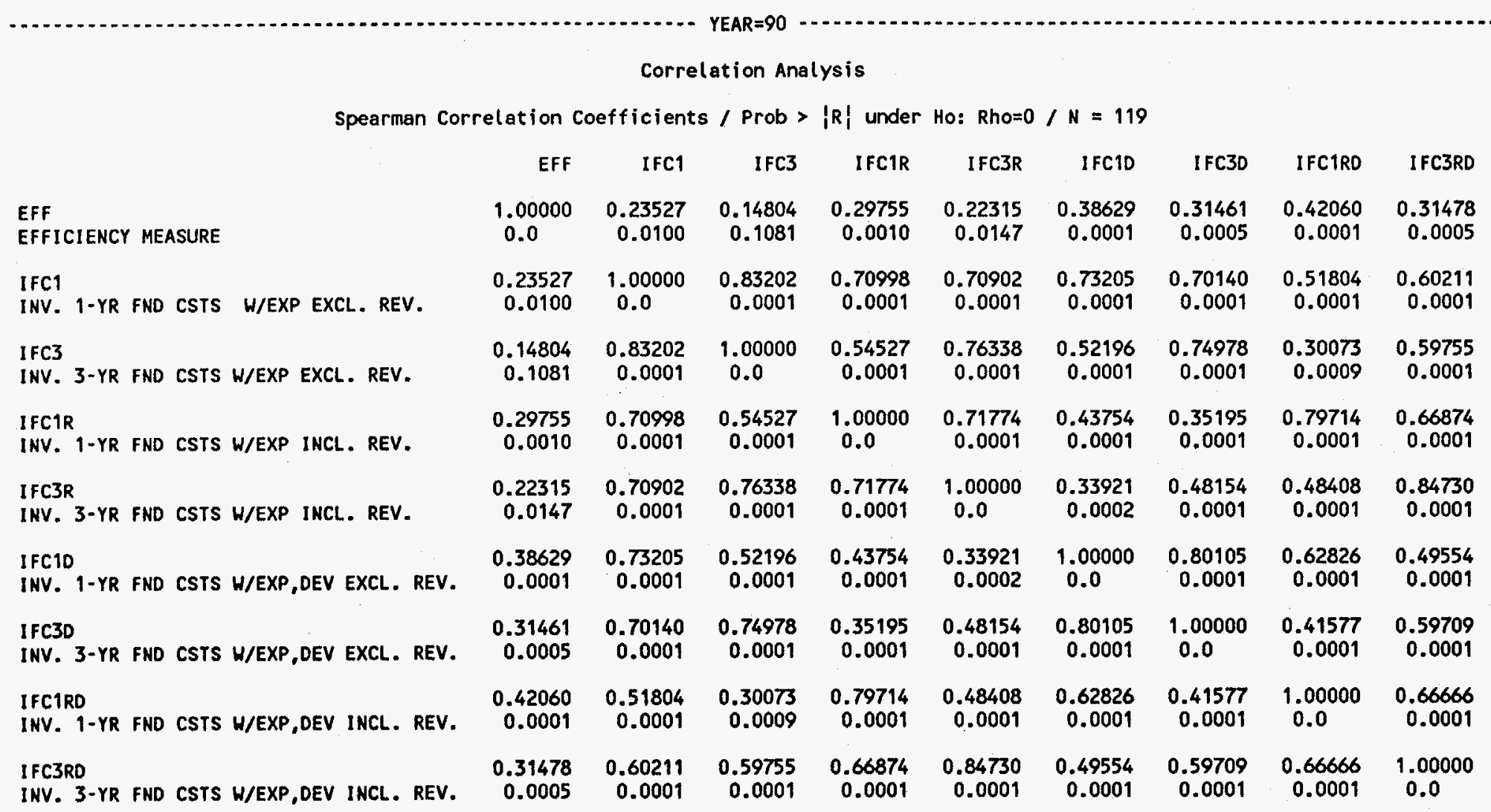


Appendix $E$

17:51 Sunday, September 25, 1994

Spearman Correlation Coefficients Between EF $F$ and Finding Costs

For Group A (Survivors) and Group C (New Entrants) - 1989-1992

Analys is By Year

\begin{tabular}{|c|c|c|c|c|c|c|c|c|}
\hline \multicolumn{9}{|c|}{ Correlation Analysis } \\
\hline & 9 'VAR' & Variables: & IFC1 & IFC3 & IFCIR & IFC1D & IFCIRD & IFC3RD \\
\hline \multicolumn{9}{|c|}{ Simple statistics } \\
\hline Variable & N & Mean & Std Dev & Median & Minimum & Maximum & Label & \\
\hline $\begin{array}{l}\text { EFF } \\
\text { IFC1 } \\
\text { IFCS } \\
\text { IFC1R } \\
\text { IFC3R } \\
\text { IFC1D } \\
\text { IFC30 } \\
\text { IFC1RD } \\
\text { IFC3RD }\end{array}$ & $\begin{array}{l}119 \\
119 \\
119 \\
119 \\
119 \\
119 \\
119 \\
119 \\
119\end{array}$ & $\begin{array}{l}0.26993 \\
0.55615 \\
0.58532 \\
0.57925 \\
0.73327 \\
0.16038 \\
0.16755 \\
0.15463 \\
0.19915\end{array}$ & $\begin{array}{l}0.18762 \\
0.73075 \\
0.56698 \\
1.26608 \\
0.89182 \\
0.15317 \\
0.09293 \\
0.29954 \\
0.13745\end{array}$ & $\begin{array}{l}0.25100 \\
0.34801 \\
0.39926 \\
0.33244 \\
0.50094 \\
0.11336 \\
0.14516 \\
0.13116 \\
0.17352\end{array}$ & $\begin{array}{r}0.00873 \\
0.02671 \\
0.03811 \\
-3.54386 \\
-0.09482 \\
0.00490 \\
0.01202 \\
-0.79216 \\
-0.06854\end{array}$ & $\begin{array}{l}1.00000 \\
6.10383 \\
3.27976 \\
9.18557 \\
6.21837 \\
0.93860 \\
0.46598 \\
2.10794 \\
0.86408\end{array}$ & $\begin{array}{l}\text { EFFICIENCY MEASURE } \\
\text { INV. } 1-Y R \text { FND CSTS } \\
\text { INV. 3-YR FND CSTS } \\
\text { INV. } 1-Y R \text { FND CSTS } \\
\text { INV. 3-YR FND CSTS } \\
\text { INV. } 1-Y R \text { FND CSTS } \\
\text { INV. 3-YR FND CSTS } \\
\text { INV. } 1-Y R \text { FND CSTS } \\
\text { INV. 3-YR FND CSTS }\end{array}$ & $\begin{array}{l}\text { W/EXP EXCL. REV. } \\
\text { W/EXP EXCL. REV. } \\
\text { W/EXP INCL. REV. } \\
\text { W/EXP INCL. REV. } \\
\text { W/EXP, DEV EXCL. REV. } \\
\text { W/EXP,DEV EXCL. REV. } \\
\text { W/EXP,DEV INCL. REV. } \\
\text { W/EXP,DEV INCL. REV. }\end{array}$ \\
\hline
\end{tabular}


Appendix E

17:51 Sunday, September 25, $1994 \quad 44$

Spearman Correlation Coefficients Between EFF and Finding Costs

For Group A (Survivors) and Group C (New Entrants) - 1989-1992

Analysis By Year

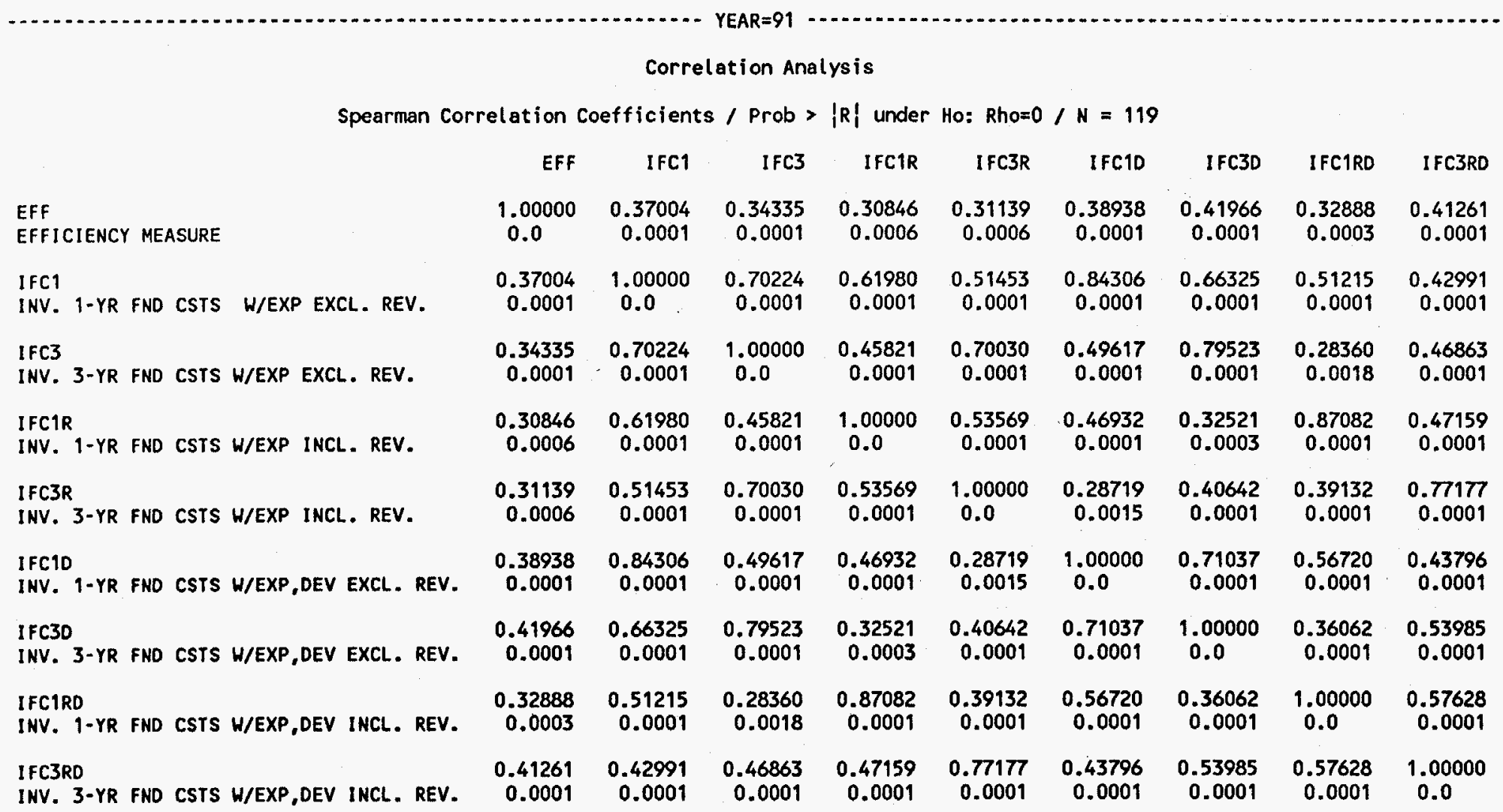


Appendix E

17:51 Sunday, September 25, 199445

Spearman Correlation Coefficients Between EFF and Finding Costs

For Group A (Survivors) and Group C (New Entrants) - 1989-1992

Analys is By Year

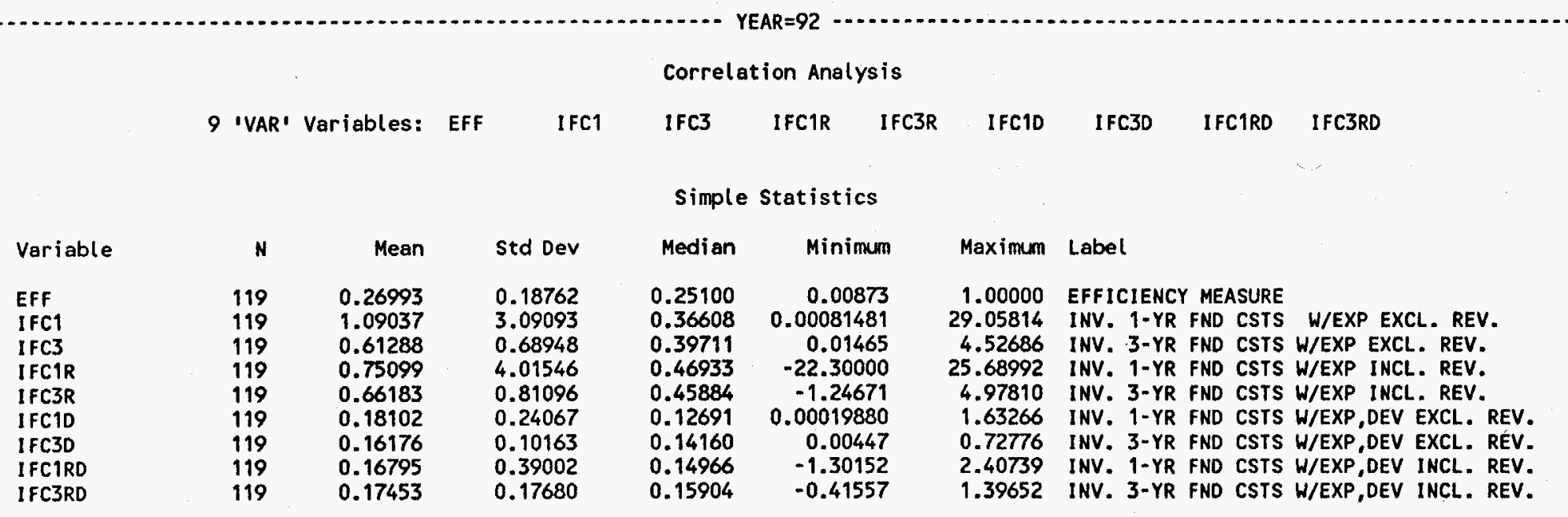


Appendix E

17:51 Sunday, September 25, 199446

Spearman Correlation Coefficients Between EFF and Finding Costs

For Group A (Survivors) and Group C (New Entrants) - 1989-1992

Analysis By Year

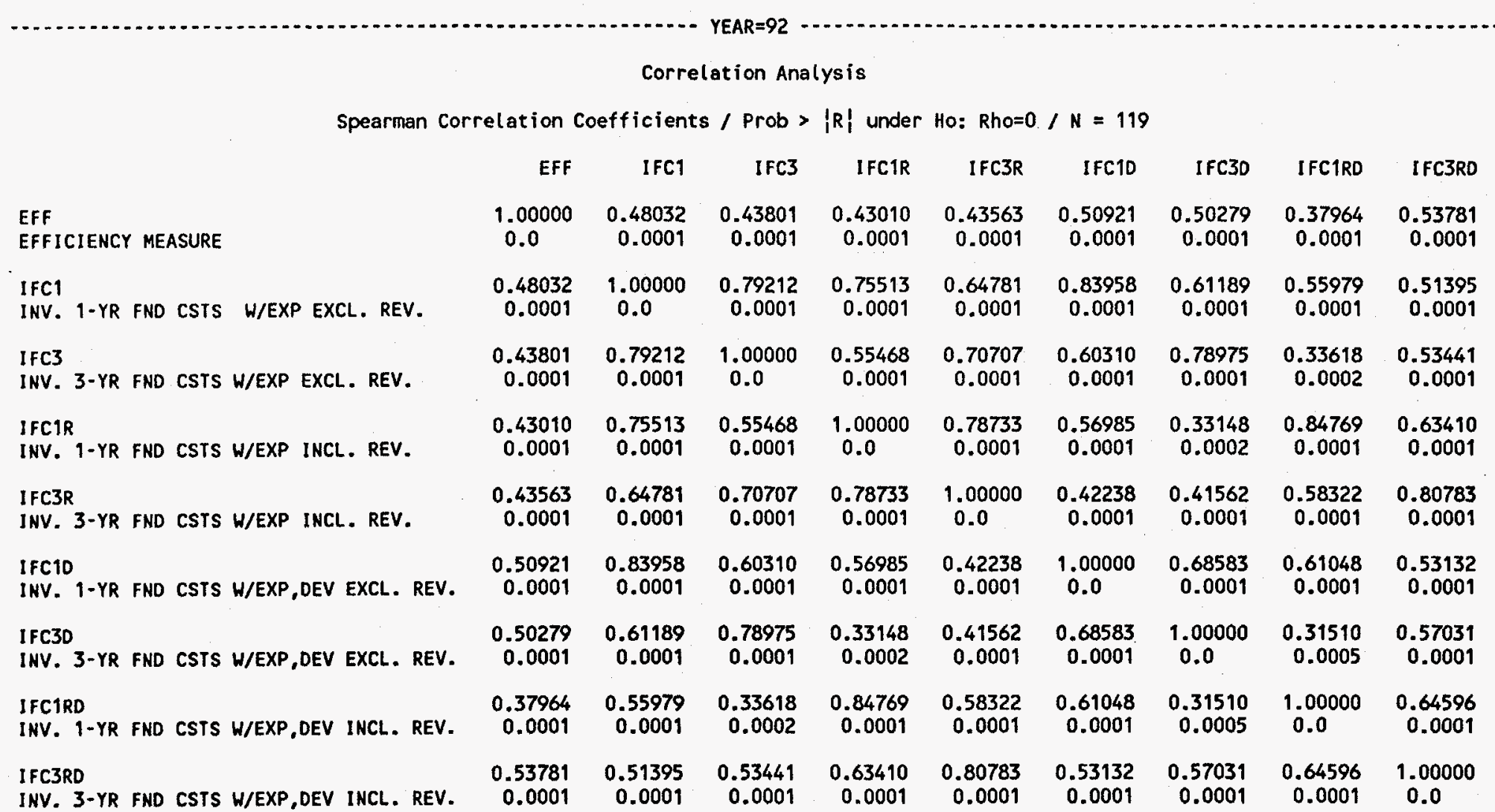


Appendix E

17:51 Sunday, September 25, 1994

Pearson Correlation Coefficients Between EFF and Finding Costs

For Group A (Survivors) and Group B (Non-survivors) - 1982-1985

Analysis By Year

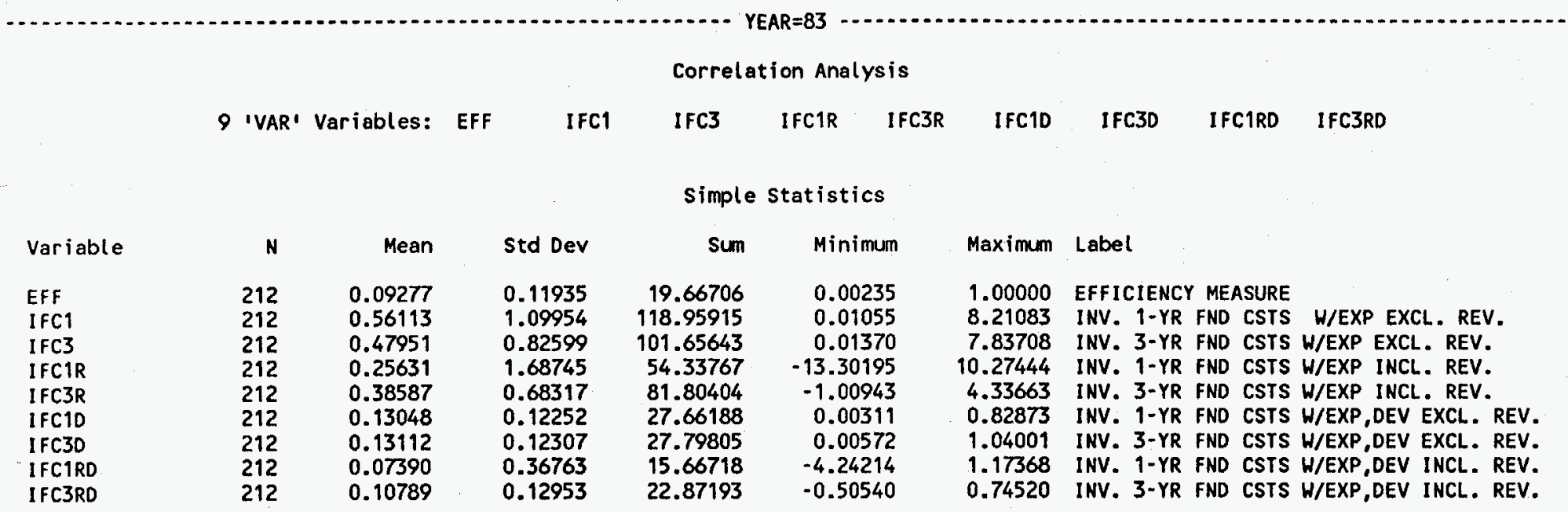


Appendix E

Pearson Correlation Coefficients Between EFF and Finding Costs

For Group A (Survivors) and Group B (Non-survivors) - 1982-1985

Analysis By Year

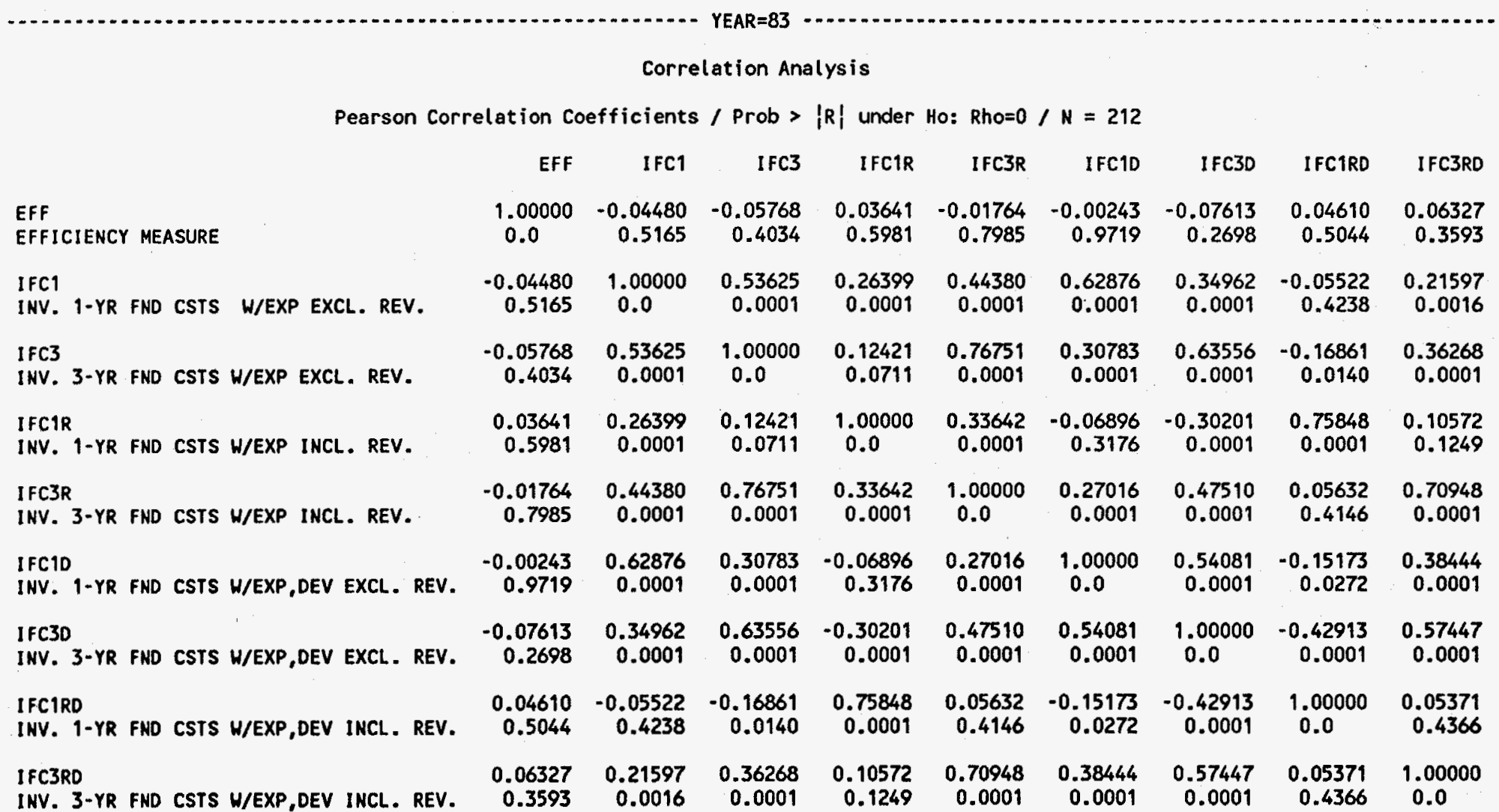


Appendix E

17:51 Sunday, September 25, 199449

Pearson Correlation Coefficients Between EFF and Finding costs

For Group A (Survivors) and Group B (Non-survivors) - 1982-1985

Analysis By Year

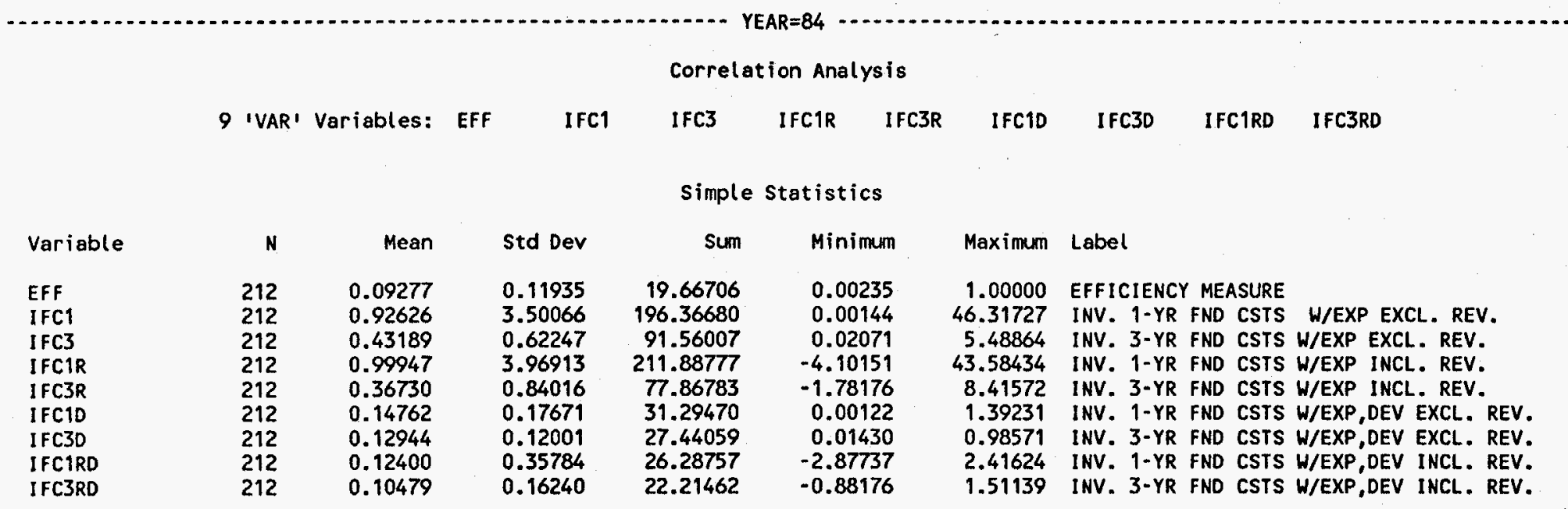


Appendix $E$

$17: 51$ Sunday, September $25,1994 \quad 50$

Pearson Correlation Coefficients Between EFF and Finding Costs

for Group A (Survivors) and Group B (Non-survivors) - 1982-1985

Analysis By Year

\section{Correlation Analysis}

Pearson Correlation Coefficients / Prob > $|R|$ under Ho: Rho=0 / N $=212$

\section{EFF}

\section{EFFICIENCY MEASURE}

IFC1

INV. 1-YR FND CSTS W/EXP EXCL. REV.

IFC3

INV. 3-YR FND CSTS W/EXP EXCL. REV.

IFCIR

INV. 1-YR FNO CSTS W/EXP INCL. REV.

IFC3R

INV. 3-YR FND CSTS W/EXP INCL. REV.

IFC10

INV. 1-YR FND CSTS W/EXP, DEV EXCL. REV.

IFC3D

INV. 3-YR FND CSTS W/EXP, DEV EXCL. REV.

IFCIRD

INV. 1-YR FND CSTS W/EXP,DEV INCL. REV.

IFC3RD

INV. 3-YR FND CSTS W/EXP,DEV INCL. REV.
EFF

IFC1

IFC3

$1.00000-0.01015$

$0.0 \quad 0.8832$

$-0.02399$

-0.02399
0.7284

IFC1R

IFC3R

IFC1D

IFC3D

IFCIRD

I FC3RD

$-0.01015$

0.8832

1.00000

0.37129

$-0.00996$

0.05529

-0.02189
0.7513

$-0.03427$

0.06490

0.06490
0.3470

0.11860

$\begin{array}{lll}0.0 & 0.0001 & 0.0001\end{array}$

0.30028

0.37228

0.22175

$\begin{array}{lllll}-0.02399 & 0.37129 & 1.00000 & 0.37220\end{array}$

0001

0001

0.0001

0.0012

0.25138

0.0001

0.0

0.0001

0.77231
0.0001

0.0001

0.79812
0.0001

0.22125

$-0.00996$

0.88415

0.37220

1.00000

0.44740

0.34921

0.26484

$0.05529 \quad 0.30028$

0.4232

0.30028
0.0001

0.77231

0.44740

0.0001

0.0001

0.0001

0.42300

0.0001

$-0.02189$

0.7513

0.37228

0.0001

0.0001

1.00000
0.0

0.39873

0.50332

0.48416

0.36156

0.0001

0.0001

0.0001

0.78502

0.78502
0.0001

$-0.03427$

$0.0001 \quad 0.0001$

0.34921
0.0001

0.39873

1.00000
0.0

0.54134
0.0001

0.55748

0.0001

0.38091

0.79812

0.26484

0.50332

0.54134

1.00000

0.09548

0.1660

0.47925

$0.06490 \quad 0.22175 \quad 0.22125 \quad 0.42300$

0,48416

1.00000

0.0001

$\begin{array}{rr}0.06490 & 0.22175 \\ 0.3470 & 0.0012\end{array}$

0.22125
0.0012

0.0001

0.0001

0.0001

0.1660

0.0

0.59210

$\begin{array}{llll}0.11860 & 0.25138 & 0.52477 & 0.36156\end{array}$

0.78502

0.38091

0.47925

0.59210

0.0001

$0.0002 \quad 0.0001$

0.0001

0.0001

0.0001

0.0 
Appendix $E$

17:51 Sunday, September 25, 199451

Pearson Correlation Coefficients Between EFF and Finding Costs

For Group A (Survivors) and Group B (Non-survivors) - 1982-1985

Analysis By Year

YEAR $=85$

Correlation Analysis

9 'VAR' Variables: EF

IFC1

IFC3

IFC1R

IFC3R

IFC1D

IFC3D IFCIRD IFC3RD

simple Statistics

$\begin{array}{lrrrrr}\text { Variable } & \text { N } & \text { Mean } & \text { Std Dev } & \text { Sum } & \text { Minimum } \\ \text { EFF } & & & & & \\ \text { IFC1 } & 212 & 0.09277 & 0.11935 & 19.66706 & 0.00235 \\ \text { IFC3 } & 212 & 1.72645 & 9.54709 & 366.00836 & 0.01033 \\ \text { IFC1R } & 212 & 0.61468 & 1.68379 & 130.31130 & 0.01471 \\ \text { IFC3R } & 212 & -0.10328 & 11.01940 & -21.89595 & -130.15385 \\ \text { IFC1D } & 212 & 0.29939 & 1.59644 & 63.47174 & -9.67640 \\ \text { IFC3D } & 212 & 0.17775 & 0.50872 & 37.68258 & 0.00292 \\ \text { IFC1RD } & 212 & 0.13471 & 0.12609 & 28.55935 & 0.00989 \\ \text { IFC3RD } & 212 & 0.02489 & 0.73174 & 5.27585 & -6.86338 \\ & 212 & 0.07157 & 0.27396 & 15.17387 & -2.34284\end{array}$

Maximum Label

1.00000 EFFICIENCY MEASURE

128.50000 INV. 1-YR FND CSTS W/EXP EXCL. REV.

20.70370 INV. 3-YR FND CSTS W/EXP EXCL. REV.

66.08333 INV. 1-YR FND CSTS W/EXP INCL. REV.

13.60435 INV. 3-YR FND CSTS W/EXP INCL. REV

6.94595 INV. 1-YR FND CSTS W/EXP, DEV EXCL. REV

1.16823 INV. 3-YR FND CSTS W/EXP,DEV EXCL. REV

3.57207 INV. 1-YR FND CSTS W/EXP,DEV INCL. REV

1.32697 INV. 3-YR FND CSTS W/EXP,DEV INCL. REV. 
Appendix $E$

Pearson Correlation Coefficients Between EFF and Finding Costs

For Group A (Survivors) and Group B (Non-survivors) - 1982-1985

Analys is By Year

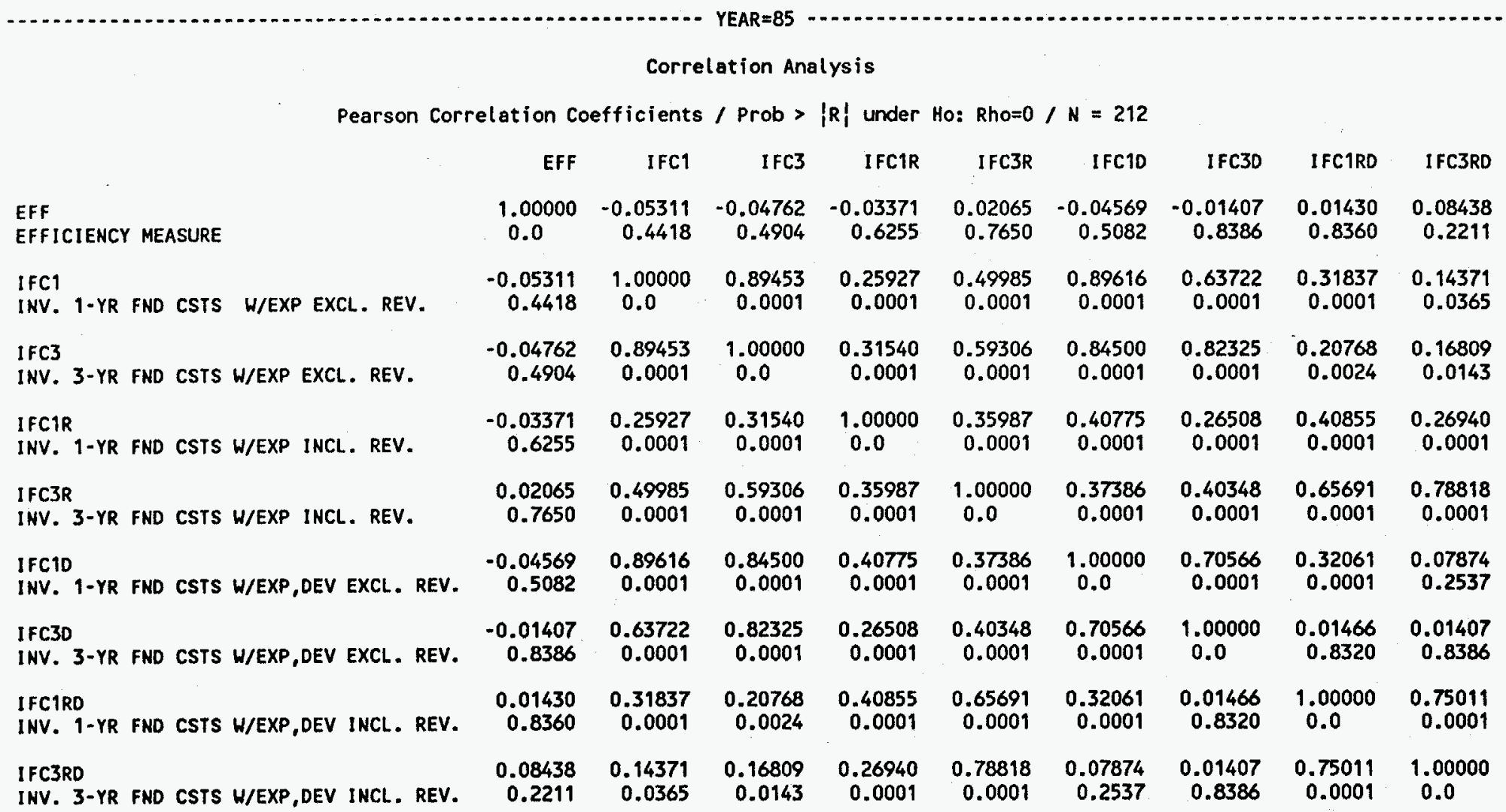


Appendix E

17:51 Sunday, September 25, 199453

Pearson Correlation Coefficients Between EFF and Finding Costs

For Group A (Survivors) and Group C (New Entrants) - 1989-1982

Analys is By Year

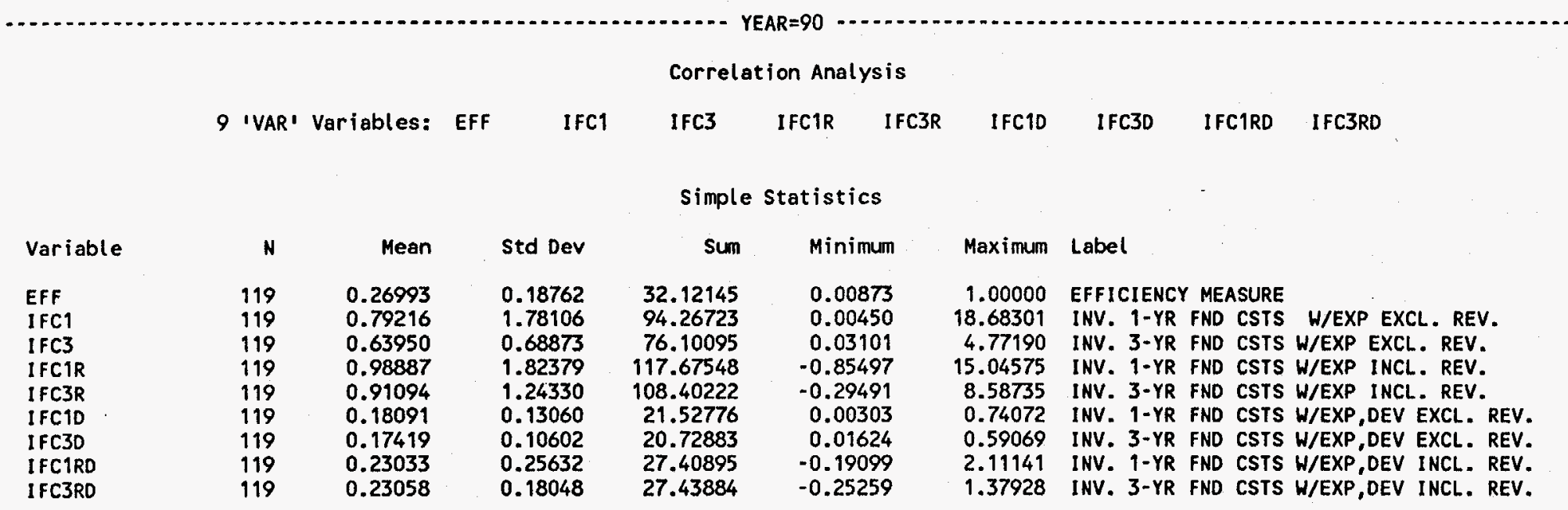


Appendix E

$17: 51$ Sunday, September 25, $1994 \quad 54$ Pearson Correlation Coefficients Between EFF and Finding costs

For Group A (Survivors) and Group C (New Entrants) - 1989-1992

Analysis By Year

YEAR $=90$

Correlation Analysis

Pearson Correlation Coefficients / Prob > $|R|$ under Ho: Rho=0 / $N=119$

EFFICIENCY MEASURE

IFC1

INV. 1-YR FND CSTS W/EXP EXCL. REV.

IFC3

INV. 3-YR FND CSTS W/EXP EXCL. REV.

IFCIR

INV. 1-YR FND CSTS W/EXP INCL. REV.

IFC3R

INV. 3-YR FND CSTS W/EXP INCL. REV.

IFC1D

INV. 1-YR FND CSTS H/EXP,DEV EXCL. REV.

IFC3D

INV. 3-YR FND CSTS W/EXP,DEV EXCL. REV.

IFCIRD

INV. 1-YR FND CSTS W/EXP,DEV INCL. REV.

IFC3RD

INV. 3-YR FND CSTS W/EXP,DEV INCL. REV.

$\begin{array}{rrrrrrrrrr}\text { EFF } & \text { IFC1 } & \text { IFC3 } & \text { IFC1R } & \text { IFC3R } & \text { IFC1D } & \text { IFC3D } & \text { IFC1RD } & \text { IFC3RD } \\ 1.00000 & 0.07415 & 0.02596 & 0.12791 & -0.04535 & 0.30355 & 0.27895 & 0.28620 & 0.23459 \\ 0.0 & 0.4229 & 0.7793 & 0.1657 & 0.6243 & 0.0008 & 0.0021 & 0.0016 & 0.0102 \\ 0.07415 & 1.00000 & 0.45130 & 0.85302 & 0.27218 & 0.34196 & 0.29121 & 0.13439 & 0.17023 \\ 0.4229 & 0.0 & 0.0001 & 0.0001 & 0.0027 & 0.0001 & 0.0013 & 0.1451 & 0.0642 \\ 0.02596 & 0.45130 & 1.00000 & 0.34623 & 0.73516 & 0.26437 & 0.53012 & 0.02905 & 0.31436 \\ 0.7793 & 0.0001 & 0.0 & 0.0001 & 0.0001 & 0.0037 & 0.0001 & 0.7538 & 0.0005 \\ 0.12791 & 0.85302 & 0.34623 & 1.00000 & 0.33983 & 0.27269 & 0.21555 & 0.47436 & 0.41800 \\ 0.1657 & 0.0001 & 0.0001 & 0.0 & 0.0002 & 0.0027 & 0.0186 & 0.0001 & 0.0001 \\ 0.04535 & 0.27218 & 0.73516 & 0.33983 & 1.00000 & 0.05193 & 0.21058 & 0.20262 & 0.53373 \\ 0.6243 & 0.0027 & 0.0001 & 0.0002 & 0.0 & 0.5748 & 0.0215 & 0.0271 & 0.0001 \\ 0.30355 & 0.34196 & 0.26437 & 0.27269 & 0.05193 & 1.00000 & 0.77435 & 0.36403 & 0.35796 \\ 0.0008 & 0.0001 & 0.0037 & 0.0027 & 0.5748 & 0.0 & 0.0001 & 0.0001 & 0.0001 \\ 0.27895 & 0.29121 & 0.53012 & 0.21555 & 0.21058 & 0.77435 & 1.00000 & 0.22638 & 0.41799 \\ 0.0021 & 0.0013 & 0.0001 & 0.0186 & 0.0215 & 0.0001 & 0.0 & 0.0133 & 0.0001 \\ 0.28620 & 0.13439 & 0.02905 & 0.47436 & 0.20262 & 0.36403 & 0.22638 & 1.00000 & 0.81363 \\ 0.0016 & 0.1451 & 0.7538 & 0.0001 & 0.0271 & 0.0001 & 0.0133 & 0.0 & 0.0001 \\ 0.23459 & 0.17023 & 0.31436 & 0.41800 & 0.53373 & 0.35796 & 0.41799 & 0.81363 & 1.00000 \\ 0.0102 & 0.0642 & 0.0005 & 0.0001 & 0.0001 & 0.0001 & 0.0001 & 0.0001 & 0.0\end{array}$


Appendix E

17:51 Sunday, September 25, 199455

Pearson Correlation Coefficients Between EFF and Finding Costs

For Group A (Survivors) and Group C (New Entrants) - 1989-1992

Analys is By Year

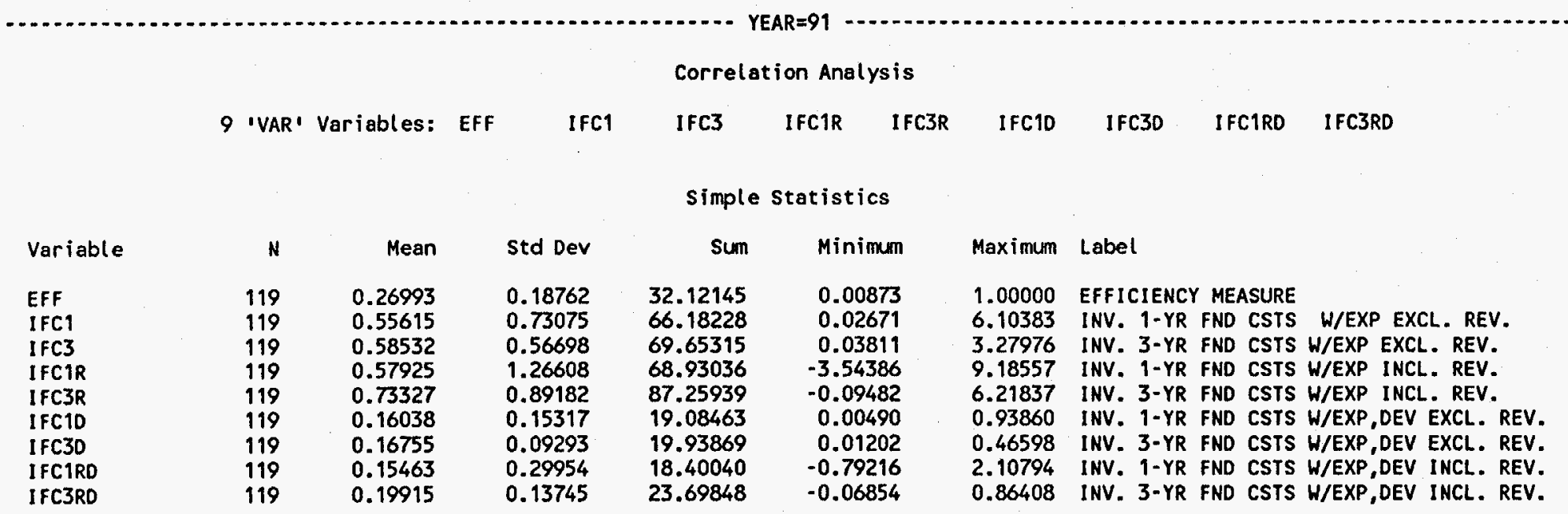


Appendix $E$

$17: 51$ sunday, September 25, 199456

Pearson Correlation Coefficients Between EFF and Finding costs

For Group A (Survivors) and Group C (New Entrants) - 1989-1992

Analysis By Year

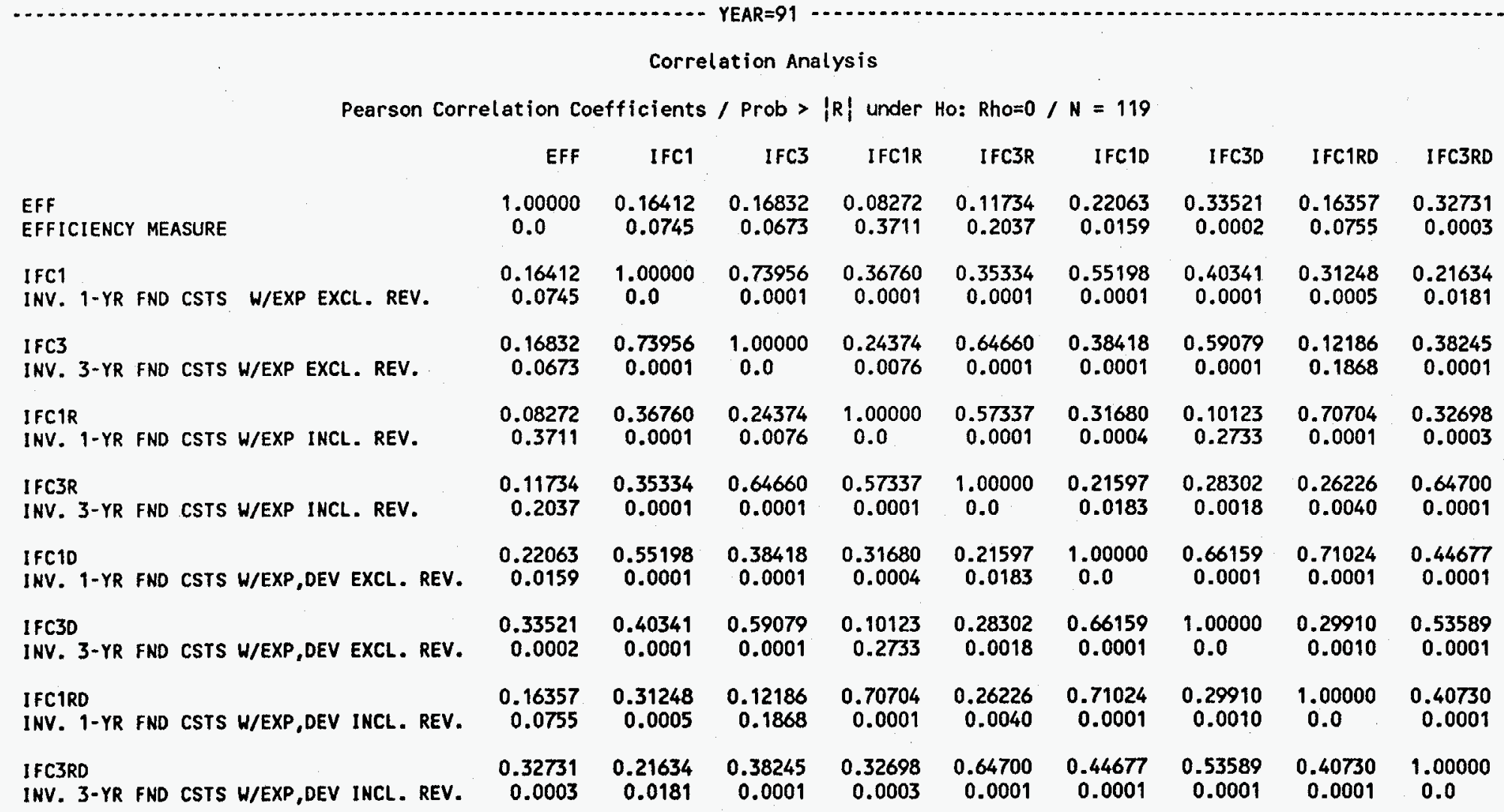


Appendix E

17:51 Sunday, September 25, 199457

Pearson Correlation Coefficients Between EFF and Finding Costs

For Group A (Survivors) and Group C (New Entrants) - 1989-1992

Analysis By Year

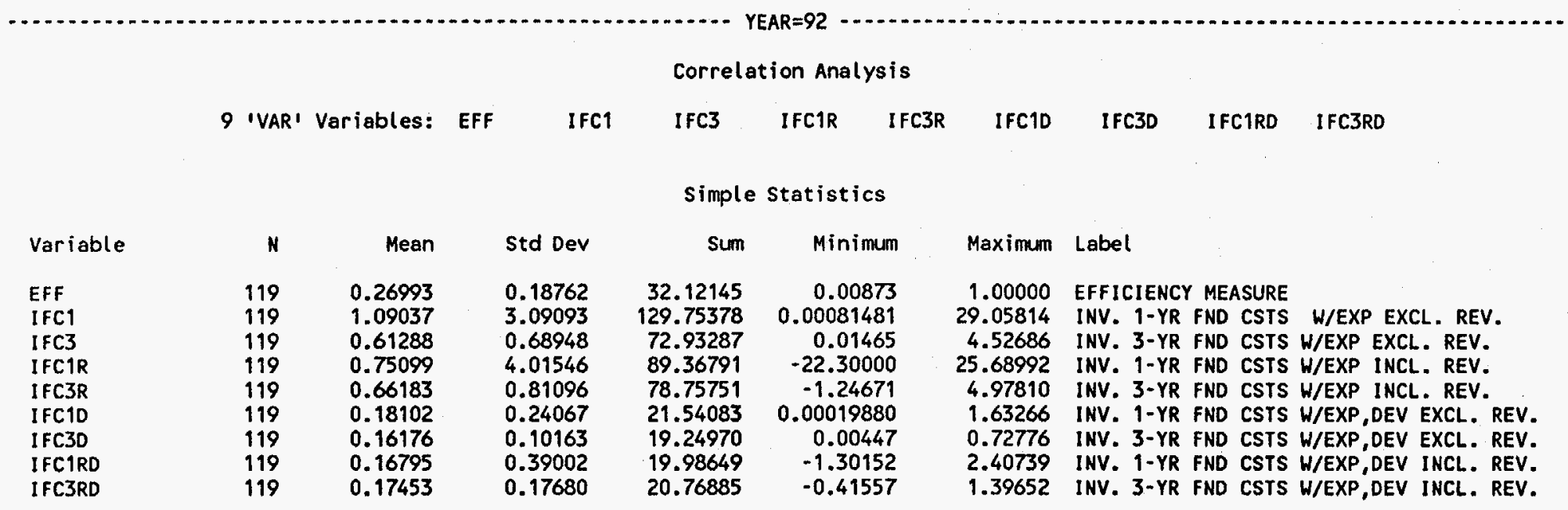


Appendix $E$

17:51 Sunday, September 25, 199458

Pearson Correlation Coefficients Between EFF and Finding costs

For Group A (Survivors) and Group C (New Entrants) - 1989-1992

Analysis By Year

\begin{tabular}{|c|c|c|c|c|c|c|c|c|c|}
\hline \multicolumn{10}{|c|}{ Correlation Analysis } \\
\hline \multicolumn{10}{|c|}{ Pearson Correlation Coefficients / Prob > $|R|$ under Ho: Rho=0 / N $=119$} \\
\hline & EFF & IFCI & IFC3 & IFCIR & IFC3R & IFC10 & $1 F C 3 D$ & IFCIRD & IFC3RD \\
\hline $\begin{array}{l}\text { EFF } \\
\text { EFFICIENCY MEASURE }\end{array}$ & $\begin{array}{l}1.00000 \\
0.0\end{array}$ & $\begin{array}{r}0.11410 \\
0.2166\end{array}$ & $\begin{array}{r}0.24249 \\
0.0079\end{array}$ & $\begin{array}{r}0.18747 \\
0.0412\end{array}$ & $\begin{array}{r}0.30100 \\
0.0009\end{array}$ & $\begin{array}{r}0.29080 \\
0.0013\end{array}$ & $\begin{array}{r}0.43270 \\
0.0001\end{array}$ & $\begin{array}{r}0.29145 \\
0.0013\end{array}$ & $\begin{array}{r}0.38188 \\
0.0001\end{array}$ \\
\hline $\begin{array}{l}\text { IFC1 } \\
\text { INV. 1-YR FND CSTS H/EXP EXCL. REV. }\end{array}$ & $\begin{array}{r}0.11410 \\
0.2166\end{array}$ & $\begin{array}{l}1.00000 \\
0.0\end{array}$ & $\begin{array}{r}0.71958 \\
0.0001\end{array}$ & $\begin{array}{r}0.62977 \\
0.0001\end{array}$ & $\begin{array}{r}0.47853 \\
0.0001\end{array}$ & $\begin{array}{r}0.61262 \\
0.0001\end{array}$ & $\begin{array}{r}0.46859 \\
0.0001\end{array}$ & $\begin{array}{r}0.35646 \\
0.0001\end{array}$ & $\begin{array}{r}0.24363 \\
0.0076\end{array}$ \\
\hline $\begin{array}{l}\text { IFC3 } \\
\text { INV. 3-YR FND CSTS W/EXP EXCL. REV. }\end{array}$ & $\begin{array}{r}0.24249 \\
0.0079\end{array}$ & $\begin{array}{r}0.71958 \\
0.0001\end{array}$ & $\begin{array}{l}1.00000 \\
0.0\end{array}$ & $\begin{array}{r}0.48296 \\
0.0001\end{array}$ & $\begin{array}{r}0.72890 \\
0.0001\end{array}$ & $\begin{array}{r}0.61230 \\
0.0001\end{array}$ & $\begin{array}{r}0.65564 \\
0.0001\end{array}$ & $\begin{array}{r}0.41678 \\
0.0001\end{array}$ & $\begin{array}{r}0.40462 \\
0.0001\end{array}$ \\
\hline $\begin{array}{l}\text { IFCIR } \\
\text { INV. 1-YR FND CSTS W/EXP INCL. REV. }\end{array}$ & $\begin{array}{r}0.18747 \\
0.0412\end{array}$ & $\begin{array}{r}0.62977 \\
0.0001\end{array}$ & $\begin{array}{r}0.48296 \\
0.0001\end{array}$ & $\begin{array}{l}1.00000 \\
0.0\end{array}$ & $\begin{array}{r}0.63986 \\
0.0001\end{array}$ & $\begin{array}{r}0.49391 \\
0.0001\end{array}$ & $\begin{array}{r}0.34121 \\
0.0001\end{array}$ & $\begin{array}{r}0.69688 \\
0.0001\end{array}$ & $\begin{array}{r}0.46544 \\
0.0001\end{array}$ \\
\hline $\begin{array}{l}\text { IFC3R } \\
\text { INV. 3-YR FND CSTS W/EXP INCL. REV. }\end{array}$ & $\begin{array}{r}0.30100 \\
0.0009\end{array}$ & $\begin{array}{r}0.47853 \\
0.0001\end{array}$ & $\begin{array}{r}0.72890 \\
0.0001\end{array}$ & $\begin{array}{r}0.63986 \\
0.0001\end{array}$ & $\begin{array}{l}1.00000 \\
0.0\end{array}$ & $\begin{array}{r}0.67161 \\
0.0001\end{array}$ & $\begin{array}{r}0.59731 \\
0.0001\end{array}$ & $\begin{array}{r}0.76516 \\
0.0001\end{array}$ & $\begin{array}{r}0.82016 \\
0.0001\end{array}$ \\
\hline $\begin{array}{l}\text { IFC1D } \\
\text { INV. 1-YR FNO CSTS W/EXP,DEV EXCL. REV. }\end{array}$ & $\begin{array}{r}0.29080 \\
0.0013\end{array}$ & $\begin{array}{r}0.61262 \\
0.0001\end{array}$ & $\begin{array}{r}0.61230 \\
0.0001\end{array}$ & $\begin{array}{r}0.49391 \\
0.0001\end{array}$ & $\begin{array}{r}0.67161 \\
0.0001\end{array}$ & $\begin{array}{l}1.00000 \\
0.0\end{array}$ & $\begin{array}{r}0.79509 \\
0.0001\end{array}$ & $\begin{array}{r}0.76079 \\
0.0001\end{array}$ & $\begin{array}{r}0.69596 \\
0.0001\end{array}$ \\
\hline $\begin{array}{l}\text { IFC3D } \\
\text { INV. 3-YR FND CSTS H/EXP,DEV EXCL. REV. }\end{array}$ & $\begin{array}{r}0.43270 \\
0.0001\end{array}$ & $\begin{array}{r}0.46859 \\
0.0001\end{array}$ & $\begin{array}{r}0.65564 \\
0.0001\end{array}$ & $\begin{array}{r}0.34121 \\
0.0001\end{array}$ & $\begin{array}{r}0.59731 \\
0.0001\end{array}$ & $\begin{array}{r}0.79509 \\
0.0001\end{array}$ & $\begin{array}{l}1.00000 \\
0.0\end{array}$ & $\begin{array}{r}0.55029 \\
0.0001\end{array}$ & $\begin{array}{r}0.67230 \\
0.0001\end{array}$ \\
\hline $\begin{array}{l}\text { IFCIRD } \\
\text { INV. 1-YR FND CSTS W/EXP,DEV INCL. REV. }\end{array}$ & $\begin{array}{r}0.29145 \\
0.0013\end{array}$ & $\begin{array}{r}0.35646 \\
0.0001\end{array}$ & $\begin{array}{r}0.41678 \\
0.0001\end{array}$ & $\begin{array}{r}0.69688 \\
0.0001\end{array}$ & $\begin{array}{r}0.76516 \\
0.0001\end{array}$ & $\begin{array}{r}0.76079 \\
0.0001\end{array}$ & $\begin{array}{r}0.55029 \\
0.0001\end{array}$ & $\begin{array}{l}1.00000 \\
0.0\end{array}$ & $\begin{array}{r}0.82034 \\
0.0001\end{array}$ \\
\hline $\begin{array}{l}\text { IFC3RD } \\
\text { INV. 3-YR FND CSTS H/EXP,DEV INCL. REV. }\end{array}$ & $\begin{array}{r}0.38188 \\
0.0001\end{array}$ & $\begin{array}{r}0.24363 \\
0.0076\end{array}$ & $\begin{array}{r}0.40462 \\
0.0001\end{array}$ & $\begin{array}{r}0.46544 \\
0.0001\end{array}$ & $\begin{array}{r}0.82016 \\
0.0001\end{array}$ & $\begin{array}{r}0.69596 \\
0.0001\end{array}$ & $\begin{array}{r}0.67230 \\
0.0001\end{array}$ & $\begin{array}{r}0.82034 \\
0.0001\end{array}$ & $\begin{array}{l}1.00000 \\
0.0\end{array}$ \\
\hline
\end{tabular}


Clinch, G. and J. Magliolo. 1992. Market perceptions of reserve disclosures under SFAS No. 69. The Accounting Review 67: 843-861.

Cornwell, C., P. Schmidt and R. Sickles. 1990. Production frontiers with cross-sectional and time-series variation in efficiency levels. Journal of Econometrics 46: 185-200.

Gaddis, D. H. Brock and C. Boynton. 1992. Pros, cons of techniques used to calculate oil, gas finding costs. oil and Gas Journal. June 1: 93-95.

Mayer, M. 1993. Interpreting the oil industry numbers. in The oil and Gas Industries ed. T. Petrie. Charlottesville, VA: AIMR Publications.

Randol, W. 1993. Factors affecting oil industry dynamics. in The Oil and Gas Industries ed. T. Petrie. Charlottesville, VA: AIMR Publications.

Schmidt, P. and R.C. Sickles. 1984. Production frontiers and panel data. Journal of Business and Economic statistics 2: $367-374$.

White, G., A. Sondhi, and D. Fried. 1994. The Analysis and Use of Financial statements New York: John Wiley \& Sons, Inc. 